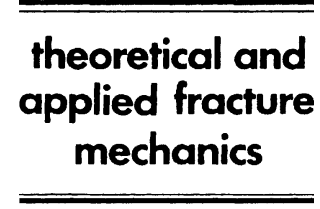

Theoretical and Applied Fracture Mechanics 38 (2002) 121-139

www.elsevier.com/locate/tafmec

\title{
Electromechanical influence of crack velocity at bifurcation for poled ferroelectric materials
}

\author{
Z.F. Song ${ }^{\text {a }}$, G.C. Sih ${ }^{\text {b,c,* }}$ \\ a Department of Engineering Mechanics, Xi'an Jiaotong University, 710049, China \\ ${ }^{\mathrm{b}}$ Institute of Mechanics, Chinese Academy of Sciences, Beijing 100080, China \\ ${ }^{\mathrm{c}}$ Department of Mechanical Engineering and Mechanics, Lehigh University, Bethlehem, PA 18015, USA
}

\begin{abstract}
The piezoelastodynamic field equations are solved to determine the crack velocity at bifurcation for poled ferroelectric materials where the applied electrical field and mechanical stress can be varied. The underlying physical mechanism, however, may not correspond to that assumed in the analytical model. Bifurcation has been related to the occurrence of a pair of maximum circumferential stress oriented symmetrically about the moving crack path. The velocity at which this behavior prevails has been referred to as the limiting crack speed.

Unlike the classical approach, bifurcation will be identified with finite distances ahead of a moving crack. Nucleation of microcracks can thus be modelled in a single formulation. This can be accomplished by using the energy density function where fracture initiation is identified with dominance of dilatation in relation to distortion. Poled ferroelectric materials are selected for this study because the microstructure effects for this class of materials can be readily reflected by the elastic, piezoelectic and dielectric permittivity constants at the macroscopic scale. Existing test data could also shed light on the trend of the analytical predictions. Numerical results are thus computed for PZT-4 and compared with those for PZT-6B in an effort to show whether the branching behavior would be affected by the difference in the material microstructures. A range of crack bifurcation speed $v_{\mathrm{b}}$ is found for different $r / a$ and $E / \sigma$ ratios. Here, $r$ and $a$ stand for the radial distance and half crack length, respectively, while $E$ and $\sigma$ for the electric field and mechanical stress. For PZT-6B with $v_{\mathrm{b}}$ in the range $100-1700 \mathrm{~m} / \mathrm{s}$, the bifurcation angles varied from $\pm 6^{\circ}$ to $\pm 39^{\circ}$. This corresponds to $E / \sigma$ of -0.072 to $0.024 \mathrm{~V} \mathrm{~m} / \mathrm{N}$. At the same distance $r / a=0.1$, PZT-4 gives $v_{\mathrm{b}}$ values of $1100-2100 \mathrm{~m} / \mathrm{s}$; bifurcation angles of $\pm 15^{\circ}$ to $\pm 49^{\circ}$; and $E / \sigma$ of -0.056 to $0.059 \mathrm{~V} \mathrm{~m} / \mathrm{N}$. In general, the bifurcation angles $\pm \theta_{0}$ are found to decrease with decreasing crack velocity as the distance $r / a$ is increased. Relatively speaking, the speed $v_{\mathrm{b}}$ and angles $\pm \theta_{0}$ for PZT- 4 are much greater than those for PZT-6B. This may be attributed to the high electromechanical coupling effect of PZT-4. Using $v_{\mathrm{b}}^{0}$ as a base reference, an equality relation $v_{\mathrm{b}}^{-}<v_{\mathrm{b}}^{0}<v_{\mathrm{b}}^{+}$can be established. The superscripts,- 0 and + refer, respectively, to negative, zero and positive electric field. This is reminiscent of the enhancement and retardation of crack growth behavior due to change in poling direction.

Bifurcation characteristics are found to be somewhat erratic when $r / a$ approaches the range $10^{-2}-10^{-1}$ where the kinetic energy densities would fluctuate and then rise as the distance from the moving crack is increased. This is an artifact introduced by the far away condition of non-vanishing particle velocity. A finite kinetic energy density prevails at infinity unless it is made to vanish in the boundary value problem.
\end{abstract}

\footnotetext{
${ }^{*}$ Corresponding author. Address: Mail Box 295, East China Univ. Science \& Technology, 130 Melong St., Shanghai 200237, China. Tel.: +86-21-64253500; fax: +86-21-64253425.

E-mail address: gcs@ecust.edu.cn (G.C. Sih).
} 
Future works are recommended to further clarify the physical mechanism(s) associated with bifurcation by means of analysis and experiment. Damage at the microscopic level needs to be addressed since it has been known to affect the macrocrack speeds and bifurcation characteristics.

(c) 2002 Published by Elsevier Science Ltd.

\section{Introduction}

Current research on dynamic crack propagation has placed great emphases on how to drive a crack beyond the Rayleigh wave speed $c_{\mathrm{R}}$, perhaps even reaching that of the dilatational wave speed $c_{\mathrm{d}}$. Such a possibility has been shown to occur in a two-dimensional molecular dynamics calculations for an in-plane shear crack moving along a weak interface dividing two harmonic crystals [1]. The shear crack is said to speed up to the Rayleigh wave velocity and then nucleate an intersonic crack travelling at the dilatational wave speed. Convincing tests, however, are lacking to explain the underlying mechanism(s). It is not known whether the Super-Rayleigh wave speed crack was accelerated from a subsonic crack or the nucleation of a new crack that could reach a speed of approximately $\sqrt{2} c_{\mathrm{s}}$ in the intersonic range. Here, $c_{\mathrm{s}}$ is the shear wave speed. Moreover, it is doubtful that local stress state is one of pure in-plane shear despite the fact that it prevails globally at distances far away from the crack tip. Should the main crack be macroscopic and the nucleated crack be microscopic [2], then there is no valid reason why the same event would not occur for an in-plane extensional crack. Equally unconvincing is the explanation offered by the finite cohesive traction shear crack model [3] where a finite peak shear stress was developed ahead of the tip. Similar models could be developed for moving cracks under in-plane extension. The solution for a finite length crack expanding at both ends with tractions can be found in [4]. The cohesive traction zones were referred to as regions of plastic yielding.

It appears that the only possible way of achieving high crack speed is to invoke a weak plane for the crack to ride. Indeed, the test in [5] enabled an in-plane extensional crack to reach the theoretical limit $c_{\mathrm{R}}$. This was accomplished by making the perspective crack path material weaker than the surrounding media. In an ordinary homogeneous isotropic solid, the limiting crack speed is about $0.5 c_{\mathrm{R}}$. Along the similar line of thought [6], an inplane shear crack was made to run along a layer of brittle polyester resin such that it was able to reach the intersonic speed of $\sqrt{2} c_{\mathrm{s}}$ and sometimes the dilatational wave speed $c_{\mathrm{d}}$. Another variation is to cut a deep groove into the specimen [7] to guide the shear crack.

The present trend is to combine the continuum approach with that of atomistic simulation where large scale numerical calculations are necessary. A case in point is the cohesive failure zone model [8] that uses the mesh-free numerical method. While the results included wavy fracture paths and crack branching, the absence of definite conclusions were attributed to the lack of an intrinsic length scale in the formulation. Scale length dependent theories, however, do not address the hierarchical character of damage by cracking. Once the length parameter is committed to a given scale, the results become restrictive. It is still necessary to know how the micro-, meso- and macroscale events are connected. There is the need to probe deeper into the continuum model of dynamic crack propagation where scaling can be addressed in a general sense. The virtue of using both the continuum and particulate approaches has been discussed $[9,10]$. Of immediate relevancy are the following:

- To include the nucleation of microcracks in relation to the propagation of a macrocrack such that failure depends on finite distances from the maincrack.

- To obtain effective account of how microstructure changes can be reflected via the macroscopic material constants. 
- To assess the hierarchical nature of failure of damage and connect the results at the micro-, meso- and macroscale.

The continuum theory of linear piezoelasticity appears to be equipped to serve the aforementioned objectives. More demanding and less apparent are the selection of a criterion that would not handicap the field solution to explain crack bifurcation. Mechanics refers to the onset of crack bifurcation as a metastable state followed by instability. The energy release rate concept though appears to be infallible for it sets an a priori that the excess energy in a system converts to the creation of fracture surface. Determination of the excess or available energy, however, cannot be bypassed by focusing attention at the crack point. It is necessary to know the energy dissipated over a finite region that would otherwise be present to trigger instability. The development of plastic enclaves prior to ductile fracture is an example. Sites of bifurcation should be identified with finite distances from the main crack. The crack-carry inertia tends to support the distant dependent character of crack bifurcation or branching. Multiple branches at a smaller length scale could also arise. Keep in mind that the kinetic energy of the particles ahead of the crack vary with the distance from the tip.

The energy density criterion is chosen because it imposes no restriction on the size regime under examination and it devoids of obvious ambiguities. For a running crack, the maximum stress is parallel to the crack [11] rather than being normal to it. This contradicts the assumption of the maximum stress criterion. The energy density approach [12-14] accounts for the influence of both stresses and strains to dilate and distort material elements. Nucleation sites of cracks are determined from the stationary values of the energy density function. Dominance of dilatation in contrast to distortion in a unit volume of material is assumed to determine the location(s) of failure by cracking rather than relying on a single stress component. This would not only discriminate the other stress components but also the strain components.

What will be shown in the works to follow are the variables that could entail crack bifurcation.
They could depend sensitively on material microstructures and external disturbances. The ultimate aim is to understand these effects and to be able to control them in application.

\section{Basic equations of piezoelastodynamics}

The constitutive equations of linear piezoelasticity are given by

$$
\sigma_{i j}=C_{i j k l} \in_{k l}-e_{k i j} E_{k}, \quad D_{i}=e_{i k l} \epsilon_{k l}+\varepsilon_{i k} E_{k}
$$

in which $\sigma_{i j}, \in_{i j}, E_{i}$ and $D_{i}$ are respectively the stress, strain, electric field and displacement. The elastic, piezoelecric and dielectric constants are given in the order of $C_{i j k l}, e_{i j k}$ and $\varepsilon_{i j}$. In plane extension with the pole aligned in the 3-direction, Eq. (1) can be simplified and written in matrix form as

$$
\left[\begin{array}{l}
\sigma_{11} \\
\sigma_{33} \\
\sigma_{31} \\
D_{1} \\
D_{3}
\end{array}\right]=\left[\begin{array}{ccccc}
c_{11} & c_{13} & 0 & 0 & e_{31} \\
c_{13} & c_{33} & 0 & 0 & e_{33} \\
0 & 0 & c_{44} & e_{15} & 0 \\
0 & 0 & e_{15} & -\epsilon_{11} & 0 \\
e_{31} & e_{33} & 0 & 0 & -\epsilon_{33}
\end{array}\right]\left[\begin{array}{l}
\epsilon_{11} \\
\epsilon_{33} \\
2 \epsilon_{31} \\
-E_{1} \\
-E_{3}
\end{array}\right]
$$

Invoked in Eq. (2) is the state of transverse anisotropy.

\subsection{Representation by potential functions}

In two-dimensions, the displacement components can be expressed in terms of the potential functions $\phi(x, y, t)$ and $\psi(x, y, t)$ :

$u_{x}=\frac{\partial \phi}{\partial x}+\frac{\partial \psi}{\partial y}, \quad u_{y}=\frac{\partial \phi}{\partial y}-\frac{\partial \psi}{\partial x}$

If $\Phi(x, y, t)$ represents the electric potential, then

$E_{x}=-\frac{\partial \Phi}{\partial x}, \quad E_{y}=-\frac{\partial \Phi}{\partial y}$

The strains are thus given by

$$
\begin{aligned}
& \epsilon_{x}=\frac{\partial^{2} \phi}{\partial x^{2}}+\frac{\partial^{2} \psi}{\partial x \partial y}, \quad \in_{y}=\frac{\partial^{2} \phi}{\partial x^{2}}-\frac{\partial^{2} \psi}{\partial x \partial y}, \\
& 2 \in_{x y}=2 \frac{\partial^{2} \phi}{\partial x \partial y}+\frac{\partial^{2} \psi}{\partial y^{2}}-\frac{\partial^{2} \psi}{\partial x^{2}}
\end{aligned}
$$


Using Eq. (2), it is found that

$$
\begin{aligned}
& \sigma_{x}=c_{11}\left(\frac{\partial^{2} \phi}{\partial x^{2}}+\frac{\partial^{2} \psi}{\partial x \partial y}\right)+c_{13}\left(\frac{\partial^{2} \phi}{\partial y^{2}}-\frac{\partial^{2} \psi}{\partial x \partial y}\right)+e_{31} \frac{\partial \Phi}{\partial y} \\
& \sigma_{y}=c_{13}\left(\frac{\partial^{2} \phi}{\partial x^{2}}+\frac{\partial^{2} \psi}{\partial x \partial y}\right)+c_{33}\left(\frac{\partial^{2} \phi}{\partial y^{2}}-\frac{\partial^{2} \psi}{\partial x \partial y}\right)+e_{33} \frac{\partial \Phi}{\partial y} \\
& \sigma_{x y}=c_{44}\left(2 \frac{\partial^{2} \phi}{\partial x \partial y}+\frac{\partial^{2} \psi}{\partial y^{2}}-\frac{\partial^{2} \psi}{\partial x^{2}}\right)+e_{15} \frac{\partial \Phi}{\partial x}
\end{aligned}
$$

Moreover, the electric displacement components become

$$
\begin{aligned}
& D_{x}=e_{15}\left(2 \frac{\partial^{2} \phi}{\partial x \partial y}+\frac{\partial^{2} \psi}{\partial y^{2}}-\frac{\partial^{2} \psi}{\partial x^{2}}\right)-\varepsilon_{11} \frac{\partial \Phi}{\partial x} \\
& D_{y}=e_{31}\left(\frac{\partial^{2} \phi}{\partial x^{2}}+\frac{\partial^{2} \psi}{\partial x \partial y}\right)+e_{33}\left(\frac{\partial^{2} \phi}{\partial y^{2}}-\frac{\partial^{2} \psi}{\partial x \partial y}\right)-\varepsilon_{33} \frac{\partial \Phi}{\partial y}
\end{aligned}
$$

The equations of motion for the stresses are

$$
\frac{\partial \sigma_{x}}{\partial x}+\frac{\partial \sigma_{x y}}{\partial y}=\rho \frac{\partial^{2} u_{x}}{\partial t^{2}}, \quad \frac{\partial \sigma_{x y}}{\partial x}+\frac{\partial \sigma_{y}}{\partial y}=\rho \frac{\partial^{2} u_{y}}{\partial t^{2}}
$$

in which $\rho$ is the mass density and $t$ the time. Substituting Eqs. (3) and (6) into Eq. (8), there results

$$
\begin{aligned}
& c_{11}\left(\frac{\partial^{3} \phi}{\partial x^{3}}+\frac{\partial^{3} \psi}{\partial x^{2} \partial y}\right)+c_{13}\left(\frac{\partial^{3} \phi}{\partial x \partial y^{2}}-\frac{\partial^{3} \psi}{\partial x^{2} \partial y}\right) \\
& +\left(e_{31}+e_{15}\right) \frac{\partial^{2} \Phi}{\partial x \partial y}+c_{44}\left(2 \frac{\partial^{3} \phi}{\partial x \partial y^{2}}+\frac{\partial^{3} \psi}{\partial y^{3}}-\frac{\partial^{3} \psi}{\partial x^{2} \partial y}\right) \\
& =\rho \frac{\partial^{2}}{\partial t^{2}}\left(\frac{\partial \phi}{\partial x}+\frac{\partial \psi}{\partial y}\right) \\
& c_{13}\left(\frac{\partial^{3} \phi}{\partial x^{2} \partial y}+\frac{\partial^{3} \psi}{\partial y^{2} \partial x}\right)+c_{33}\left(\frac{\partial^{3} \phi}{\partial y^{3}}-\frac{\partial^{3} \psi}{\partial x^{2} \partial y}\right) \\
& +e_{33} \frac{\partial^{2} \Phi}{\partial y^{2}}+e_{15} \frac{\partial^{2} \Phi}{\partial x^{2}}+c_{44}\left(2 \frac{\partial^{3} \phi}{\partial x^{2} \partial y}+\frac{\partial^{3} \psi}{\partial x \partial y^{2}}-\frac{\partial^{3} \psi}{\partial y^{3}}\right) \\
& =\rho \frac{\partial^{2}}{\partial t^{2}}\left(\frac{\partial \phi}{\partial y}-\frac{\partial \psi}{\partial x}\right)
\end{aligned}
$$

Satisfaction of

$\frac{\partial D_{x}}{\partial x}+\frac{\partial D_{y}}{\partial y}=0$

for $D_{x}$ and $D_{y}$ in Eq. (7) yields

$$
\begin{aligned}
& e_{15}\left(2 \frac{\partial^{3} \phi}{\partial x^{2} \partial y}+\frac{\partial^{3} \psi}{\partial y^{2} \partial x}-\frac{\partial^{3} \psi}{\partial x^{3}}\right)-\varepsilon_{11} \frac{\partial^{2} \Phi}{\partial x^{2}} \\
& \quad+e_{31}\left(\frac{\partial^{3} \phi}{\partial x^{2} \partial y}+\frac{\partial^{3} \psi}{\partial x \partial y^{2}}\right)+e_{33}\left(\frac{\partial^{3} \phi}{\partial y^{3}}-\frac{\partial^{3} \psi}{\partial x \partial y^{2}}\right) \\
& \quad-\varepsilon_{33} \frac{\partial^{2} \Phi}{\partial y^{2}}=0
\end{aligned}
$$

The governing Eqs. (9) and (11) are to be solved for the potential functions $\phi, \psi$ and $\Phi$ under the specified boundary conditions.

\subsection{Constant velocity problems}

For cracks moving at a constant velocity $v$, say along the $x$-axis, it is convenient to make the following transformations:

$\xi=x-v t, \quad \eta=y$

where $\xi$ and $\eta$ stand for a set of moving coordinates and $x$ and $y$ are stationary (Fig. 1). Uniform normal stress $\sigma$ and electric $E$ are applied at infinity. A crack $2 a$ is assumed to propagate at a constant length as assumed in [15]; it closes at the trailing edge by the amount it advances. This artifact implies that the trailing tip absorbs energy at the same rate as that radiated by the leading tip.
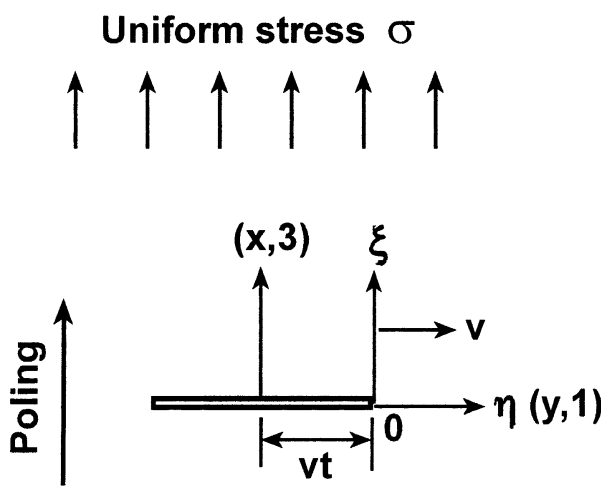

Uniform electric field E

Fig. 1. Moving coordinates and far field. 


\subsection{Galilean transformation}

The transformation in Eq. (12) can be applied to Eq. (9) whereby eliminating the time variable $t$. Let prime denote differentiation with respect to the argument of the function. The complex variable $z=\xi+\mu \eta$ with $\mu$ being a complex constant. Eqs. (9) and (11) can thus be written as

$$
\begin{aligned}
& {\left[c_{11}+\left(c_{13}+2 c_{44}\right) \mu^{2}-\rho v^{2}\right] \phi^{\prime \prime \prime}} \\
& \quad+\left[c_{44} \mu^{3}+\left(c_{11}-c_{13}-c_{44}\right) \mu-\rho v^{2} \mu\right] \psi^{\prime \prime \prime} \\
& \quad+\left(e_{31}+e_{15}\right) \mu \Phi^{\prime \prime}=0 \\
& {\left[c_{33} \mu^{3}+\left(2 c_{44}+c_{13}\right) \mu-\rho v^{2} \mu\right] \phi^{\prime \prime \prime}} \\
& \quad-\left[c_{44}-\left(c_{44}+c_{13}-c_{33}\right) \mu^{2}-\rho v^{2}\right] \psi^{\prime \prime \prime} \\
& \quad+\left(e_{15}+e_{33} \mu^{2}\right) \Phi^{\prime \prime}=0 \\
& {\left[e_{33} \mu^{3}+\left(2 e_{15}+e_{31}\right) \mu\right] \phi^{\prime \prime \prime}} \\
& \quad+\left[\left(e_{15}+e_{31}-e_{33}\right) \mu^{2}-e_{15}\right] \psi^{\prime \prime \prime} \\
& \quad-\left(\varepsilon_{11}+\varepsilon_{33} \mu^{2}\right) \Phi^{\prime \prime}=0
\end{aligned}
$$

For a non-trivial solution, the determinant of Eq. (13) is required to vanish. This gives the characteristic equation that can be solved for the eight complex roots. The pair $\pm \mathrm{i}$ can be dropped while the remaining three pairs can be solved by applying the conditions across the $x$ - or $\xi$-axis such that $\mu_{4}=\bar{\mu}_{1}, \quad \mu_{5}=\bar{\mu}_{2}, \quad \bar{\mu}_{6}=\bar{\mu}_{3}$

Bar represents conjugate. The three roots $u_{j}(j=1$, $2,3)$ can be put back into Eq. (13) for determining $\phi, \psi$ and $\Phi$.

\subsection{Complex potential functions}

Let $\alpha_{j}$ and $\beta_{j}$ represent complex constants for $j=1,2,3$ and $F_{j}\left(z_{j}\right)$ a complex function of $z_{j}=$ $\xi+\mu_{j} \eta$. It follows that

$$
\begin{aligned}
& \phi=\operatorname{Re}\left[\sum_{j=1}^{3} F_{j}\left(z_{j}\right)\right], \\
& \psi=\operatorname{Re}\left[\sum_{j=1}^{3} \alpha_{j} F_{j}\left(z_{j}\right)\right], \\
& \Phi=\operatorname{Re}\left[\sum_{j=1}^{3} \beta_{j} F_{j}\left(z_{j}\right)\right]
\end{aligned}
$$

in which $\alpha_{j}$ and $\beta_{j}$ are found from Eq. (13) once $\mu_{j}$ are known.
Inserting Eqs. (15) into (6), there results

$$
\begin{aligned}
& \sigma_{x}=\operatorname{Re}\left\{\sum _ { j = 1 } ^ { 3 } \left[c_{11}+c_{13} \mu_{j}^{2}+\left(c_{11}-c_{13}\right) \mu_{j} \alpha_{j}\right.\right. \\
&\left.\left.+e_{31} \mu_{j} \beta_{j}\right] F_{j}^{\prime \prime}\left(z_{j}\right)\right\} \\
& \sigma_{y}=\operatorname{Re}\left\{\sum _ { j = 1 } ^ { 3 } \left[c_{13}+c_{33} \mu_{j}^{2}+\left(c_{13}-c_{33}\right) \mu_{j} \alpha_{j}\right.\right. \\
&\left.\left.+e_{33} \mu_{j} \beta_{j}\right] F_{j}^{\prime \prime}\left(z_{j}\right)\right\} \\
& \sigma_{x y}=\operatorname{Re}\left\{\sum_{j=1}^{3}\left[2 c_{44} u_{j}+c_{44}\left(\mu_{j}^{2}-1\right) \alpha_{j}+e_{15} \beta_{j}\right] F_{j}^{\prime \prime}\left(z_{j}\right)\right\}
\end{aligned}
$$

Similarly, Eq. (15) can be put into Eq. (7) to render

$$
\begin{aligned}
D_{x}=\operatorname{Re} & \left\{\sum_{j=1}^{3}\left[2 e_{15} \mu_{j}+e_{15}\left(\mu_{j}^{2}-1\right) \alpha_{j}-\varepsilon_{11} \beta_{j}\right] F_{j}^{\prime \prime}\left(z_{j}\right)\right\} \\
D_{y}=\operatorname{Re} & \left\{\sum _ { j = 1 } ^ { 3 } \left[e_{31}+e_{33} \mu_{j}^{2}+\left(e_{31}-e_{33}\right) \mu_{j} \alpha_{j}\right.\right. \\
& \left.\left.-\varepsilon_{33} \mu_{j} \beta_{j}\right] F_{j}^{\prime \prime}\left(z_{j}\right)\right\}
\end{aligned}
$$

The expressions for $\sigma_{y}, \sigma_{x y}$ and $D_{y}$ in Eqs. (16) and (17) will be isolated by introducing the contractions

$$
\begin{aligned}
& A_{j}=C_{13}+C_{33} \mu_{j}^{2}+\left(C_{13}-C_{33}\right) \mu_{j} \alpha_{j}+e_{33} \mu_{j} \beta_{j} \\
& B_{j}=2 C_{44} \mu_{j}+C_{44}\left(\mu_{j}^{2}-1\right) \alpha_{j}+e_{15} \beta_{j} \\
& C_{j}=e_{31}+e_{33} \mu_{j}^{2}+\left(e_{31}-e_{33}\right) \mu_{j} \alpha_{j}-\varepsilon_{33} \mu_{j} \beta_{j}
\end{aligned}
$$

such that the boundary conditions involving $\sigma_{y}, \sigma_{x y}$ and $D_{y}$ can be expressed in simpler forms.

A suitable form of solution for $F_{j}^{\prime}\left(z_{j}\right)$ can be written as

$F_{j}^{\prime}\left(z_{j}\right)=b_{j} z_{j}+a_{j}\left(\sqrt{z_{j}\left(z_{j}+2 a\right)}-z_{j}\right)$

so that

$$
F_{j}^{\prime \prime}\left(z_{j}\right)=b_{j}+a_{j}\left(\frac{z_{j}+a}{\sqrt{z_{j}\left(z_{j}+2 a\right)}}-1\right)
$$


Remember that $z_{j}$ refers to the moving coordinates $\xi$ and $\eta$.

\subsection{Remote and crack surface boundary conditions}

Referring to Fig. 1, uniform mechanical stress $\sigma_{y}^{\infty}$ and electric field $E_{y}^{\infty}$ are specified at infinity. By letting $\left|z_{j}\right| \rightarrow \infty$ in Eq. (20), substituting the results into the appropriate expressions of Eqs. (16) and (17) and using Eq. (18), it can be shown that

$$
\begin{aligned}
& \operatorname{Re}\left[\sum_{j=1}^{3} A_{j} b_{j}\right]=\sigma_{y}^{\infty}, \quad \operatorname{Re}\left[\sum_{j=1}^{3} B_{j} b_{j}\right]=0, \\
& \operatorname{Re}\left[\sum_{j=1}^{3} C_{j} b_{j}\right]=0
\end{aligned}
$$

The second and third Eqs. (21) correspond to $\sigma_{x y}^{\infty}=0$ and $D_{y}^{\infty}=0$, respectively.

Three additional conditions can be identified with the crack surfaces being traction-free and impermeable of electric flux:

$\sigma_{y}=0, \sigma_{x y}=0, D_{y}=0$ for $-2 a<\xi<01$

This gives

$$
\begin{aligned}
& \operatorname{Re}\left\{\sum_{j=1}^{3} A_{j} b_{j}+[\mathrm{i} f(\xi)-1] A_{j} a_{j}\right\}=0 \\
& \operatorname{Re}\left\{\sum_{j=1}^{3} B_{j} b_{j}+[\mathrm{i} f(\xi)-1] B_{j} a_{j}\right\}=0 \\
& \operatorname{Re}\left\{\sum_{j=1}^{3} C_{j} b_{j}+[\mathrm{i} f(\xi)-1] C_{j} a_{j}\right\}=0
\end{aligned}
$$

in which the real function $f(\xi)$ takes the form:

$f(\xi)=-\frac{\xi+a}{\sqrt{-\xi(\xi+2 a)}}$, for $-2 a<\xi<0$

Eqs. (22) determine the constants $a_{j}(j=1,2,3)$ while $b_{j}(j=1,2,3)$ can be found from Eq. (21), $E_{x}^{\infty}=0$ and $E_{y}^{\infty}$ specified in addition to zero rotation at infinity. Hence, Eqs. (15) are completely known from which all the stresses, electric fields and displacements can be found.
A physical requirement is that the crack surface normal displacement $u_{y}$ is required to be positive. Eqs. (15) and (19) can be used to express the second of Eq. (3) as

$u_{y}=\operatorname{Re}\left\{\sum_{j=1}^{3}\left(\mu_{j}-\alpha_{j}\right)\left(b_{j} z_{j}+a_{j} \sqrt{z_{j}\left(z_{j}+2 a\right)}-a_{j} z_{j}\right)\right\}$

For a given crack velocity and electromechanical disturbance $E_{y}^{\infty} / \sigma_{y}^{\infty}$, the condition $u_{y}>0$ must be satisfied for $-2 a<\xi<0$ and $\eta=0$ so that the upper and lower crack surfaces would not come in contact.

\subsection{Energy density functions}

The energy density function $\mathrm{d} W / \mathrm{d} V$ is positive definite and can be calculated from

$\frac{\mathrm{d} W}{\mathrm{~d} V}=\frac{1}{2} \sigma_{i j} \in_{i j}+\frac{1}{2} D_{i} E_{i}$

in which $\sigma_{x}, \sigma_{y}$ and $\sigma_{x y}$ are given in Eq. (16) and $D_{x}$, $D_{y}$ in Eq. (17). Note that $\in_{x}, \in_{y}, \in_{x y}, E_{x}$ and $E_{y}$ in Eqs. (5) and (4) can be found from $\phi, \psi$ and $\Phi$ in Eq. (15). These expressions are solved numerically for $\mathrm{d} W / \mathrm{d} V$ in Eq. (26).

\section{Crack bifurcation criterion}

A possible explanation for crack bifurcation has been attributed to the appearance of maximum circumferential stress on planes inclined at about $\pm 60^{\circ}$ to the direction of a crack moving faster than $0.6 c_{\mathrm{s}}$ [15]. A variety of criteria have since been used; they represent different variations of the maximum stress concept. The predicted angles of bifurcation, however, are found to be much larger than those observed in tests. The energy release rate for multiple branching spreading in a circular pattern has been estimated in $[16,17]$. However, no quantitative assessments of possible effects due to material properties, boundary conditions and microcracking have been made.

The volume energy density approach was first used in [11] where attention were focused on the failure of an material element at a finite distance 
ahead of the tip. An asymptotic field solution $\mathrm{d} W / \mathrm{d} V=S / r$ with $r \rightarrow 0$ was made use of. Bifurcation angles of $\pm 18.84^{\circ}$ to $\pm 15.52^{\circ}$ were reported for glass with Poisson's ratio of 0.21-0.24. The assumption was based on finding a pair of radial planes off to the main path. On these planes, $S$ were found to possess relative minima. This corresponds to the largest value(s) of dilatational energy density exceeding that ahead of the main crack prior to branching. No precise information were given for the distance at which bifurcation occurred. It remains unclear how the asymptotic decay $S / r$ could be used to discuss failure that is initiated at a finite distance from the crack tip. Comparison of the asymptotic solution with the full field solution of $\mathrm{d} W / \mathrm{d} V$ for a stationary crack has been made in [18]. The difference can be sig- nificant for examining damage inflicted to the material ahead of the main crack.

\subsection{Numerical procedure}

To be specific, numerical results will be calculated for PZT-4 whose material properties can be found in Table 1. According to Eq. (13), the crack velocity must be specified before the roots $\mu_{j}$ $(j=1,2,3)$ can be determined. Table 2 gives $u_{j}$ for crack velocities of $v=400,1300$ and $1800 \mathrm{~m} / \mathrm{s}$. No appreciable changes take place until the high crack velocity range is reached. The corresponding values of $\alpha_{j}$ and $\beta_{j}$ in Eq. (15) for the same crack velocities are given in Table 3 . For illustration, it suffices to discuss the case for $v=$ $1300 \mathrm{~m} / \mathrm{s}$.

Table 1

Material constants for PZT-4 piezoceramic

\begin{tabular}{|c|c|c|c|c|c|c|c|c|}
\hline \multicolumn{4}{|c|}{ Elastic constants $\times 10^{10}\left(\mathrm{~N} / \mathrm{m}^{2}\right)$} & \multicolumn{3}{|c|}{ Piezoelectric constants $\left(\mathrm{C} / \mathrm{m}^{2}\right)$} & \multicolumn{2}{|c|}{$\begin{array}{l}\text { Dielectric } \\
\text { permittivities } \times 10^{-9}(\mathrm{C} / \mathrm{V} \mathrm{m})\end{array}$} \\
\hline$c_{11}$ & $c_{13}$ & $c_{33}$ & $c_{44}$ & $e_{31}$ & $e_{33}$ & $e_{15}$ & $\varepsilon_{11}$ & $\varepsilon_{33}$ \\
\hline 13.9 & 7.43 & 11.3 & 2.56 & -6.98 & 13.84 & 13.44 & 6.00 & 5.47 \\
\hline
\end{tabular}

Table 2

Roots for PZT-4 with specified crack velocities

\begin{tabular}{llll}
\hline Roots & \multicolumn{2}{l}{ Crack velocities $(\mathrm{m} / \mathrm{s})$} & 1800 \\
\cline { 2 - 4 } & 400 & 1300 & $(0.00+1.158 \mathrm{i})$ \\
$\mu_{1}$ & $(0.00+1.189 \mathrm{i})$ & $(0.00+1.173 \mathrm{i})$ & $(0.376+0.876 \mathrm{i})$ \\
$\mu_{2}$ & $(0.281+1.078 \mathrm{i})$ & $(0.337+0.985 \mathrm{i})$ & $(-0.376+0.876 \mathrm{i})$ \\
$\mu_{3}$ & $(-0.281+1.078 \mathrm{i})$ & $(-0.337+0.985 \mathrm{i})$ & \\
\hline
\end{tabular}

Table 3

Coefficients in Eq. (15) for PZT-4

\begin{tabular}{llll}
\hline Roots & Crack velocities $(\mathrm{m} / \mathrm{s})$ & \\
\cline { 2 - 4 } & 400 & 1300 & 1800 \\
\hline$\alpha_{1}$ & $(0.00-2.463 \mathrm{i})$ & $(0.00-5.816 \mathrm{i})$ & $(0.00+46.97 \mathrm{i})$ \\
$\alpha_{2}$ & $(-1.341+3.061 \mathrm{i})$ & $(-0.003+3.762 \mathrm{i})$ & $(1.512+3.816 \mathrm{i})$ \\
$\alpha_{3}$ & $(1.341+3.061 \mathrm{i})$ & $(0.003+3.762 \mathrm{i})$ & $(-1.512+3.816 \mathrm{i})$ \\
$\beta_{1}$ & $(0.00-0.448 \mathrm{i})$ & $(0.00-1.317 \mathrm{i})$ & $(0.00+12.33 \mathrm{i})$ \\
$\beta_{2}$ & $(-0.082-0.404 \mathrm{i})$ & $(-2.769-0.434 \mathrm{i})$ & $(-0.489-0.390 \mathrm{i})$ \\
$\beta_{3}$ & $(0.082-0.404 \mathrm{i})$ & $(2.769-0.434 \mathrm{i})$ & $(-0.489-0.390 \mathrm{i})$ \\
\hline
\end{tabular}




\subsection{Multiscale feature of bifurcation}

One of the objectives is to compute the energy density function from Eq. (26) and to determine the minima of $\mathrm{d} W / \mathrm{d} V$ with respect to the angle $\theta$ for negative and positive $E_{y}^{\infty} / \sigma_{y}^{\infty}$. Both single value and double values of $\theta_{0}$ will be determined. The direction(s) of dilatation dominance which is assumed to coincide with the prospective crack path. Fig. 2(a) and (b) shows the two possible solutions: single crack path $\theta_{0}=0^{\circ}$ and crack bifurcation, $\pm \theta_{0}$. The radius of the core region $r_{0}$ is small but non-zero; it prevents the energy density $\mathrm{d} W / \mathrm{d} V$ to become unbounded. Note that the results in Table 4 depend on $r / a$ ranging from $10^{-6}$ to $10^{-1}$. Unless minimum of $\mathrm{d} W / \mathrm{d} V$ for bifurcation is shown, $\theta_{0}=0^{\circ}$ is implied for a crack to run straight ahead. When $E_{y}^{\infty} / \sigma_{y}^{\infty}$ is positive $\theta_{0}=0^{\circ}$ is predicted for all values of $r / a$. The corresponding normalized energy density function $\left\{(\mathrm{d} W / \mathrm{d} V)_{\min } /\right.$ $\left.\left[a\left(\sigma_{y}^{\infty}\right)^{2}\right]\right\} \times 10^{-10}$ are summarized in Table 5 . It decreases sharply with the distance $r / a$. For

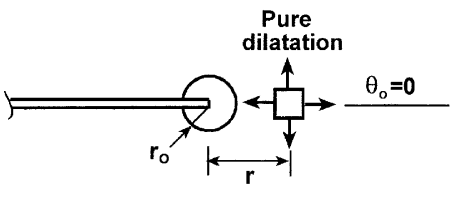

(a) Straight crack path

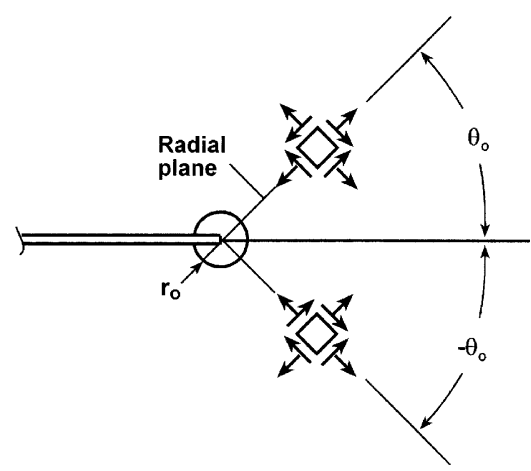

(b) Crack bifurcation

Fig. 2. Crack bifurcation assumed to coincide with dominance of dilatation.

Table 4

Prediction of crack initiation direction(s) for $v=1300 \mathrm{~m} / \mathrm{s}$ and PZT-4

\begin{tabular}{llllll}
\hline$E_{y}^{\infty} / \sigma_{y}^{\infty} \times 10^{-2}$ & $r / a$ & & & $10^{-2}$ & $10^{-1}$ \\
\cline { 2 - 5 }$(\mathrm{V} \mathrm{m} / \mathrm{N})$ & $10^{-6}$ & $10^{-4}$ & $10^{-3}$ & $\pm 69.3^{\circ}$ & $\pm 94.5^{\circ}$ \\
-8.00 & $\pm 70.2^{\circ}$ & $\pm 70.2^{\circ}$ & $\pm 70.2^{\circ}$ & $\pm 72.0^{\circ}$ & $\pm 16.2^{\circ}$ \\
-5.33 & $\pm 72.0^{\circ}$ & $\pm 72.0^{\circ}$ & $\pm 72.0^{\circ}$ & - & $0^{\circ}$ \\
-2.66 & $\pm 91.8^{\circ}$ & $96.3^{\circ}$ & - & $0^{\circ}$ & $0^{\circ}$ \\
0.00 & - & - & - & &
\end{tabular}

Table 5

Normalized minimum energy density $\left\{(\mathrm{d} W / \mathrm{d} V)_{\min } /\left[a\left(\sigma_{y}^{\infty}\right)^{2}\right]\right\} \times 10^{-10}$ for $v=1300 \mathrm{~m} / \mathrm{s}$ and PZT-4

\begin{tabular}{|c|c|c|c|c|c|}
\hline \multirow{2}{*}{$\begin{array}{l}E_{y}^{\infty} / \sigma_{y}^{\infty} \times 10^{-2} \\
(\mathrm{~V} \mathrm{~m} / \mathrm{N})\end{array}$} & \multicolumn{5}{|l|}{$r / a$} \\
\hline & $10^{-6}$ & $10^{-4}$ & $10^{-3}$ & $10^{-2}$ & $10^{-1}$ \\
\hline-8.00 & $9.772 \times 10^{4}$ & $9.763 \times 10^{2}$ & $9.748 \times 10^{1}$ & 9.763 & 1.022 \\
\hline-5.33 & $5.199 \times 10^{4}$ & $5.193 \times 10^{2}$ & $5.187 \times 10^{1}$ & 5.222 & 0.535 \\
\hline-2.66 & $3.784 \times 10^{4}$ & $3.769 \times 10^{2}$ & - & - & 0.289 \\
\hline 0.00 & - & - & - & 5.723 & 0.454 \\
\hline 2.66 & $1.189 \times 10^{5}$ & $1.177 \times 10^{3}$ & $1.150 \times 10^{2}$ & $1.082 \times 10^{1}$ & 1.030 \\
\hline 5.33 & $2.050 \times 10^{5}$ & $2.038 \times 10^{3}$ & $2.012 \times 10^{2}$ & $1.954 \times 10^{1}$ & 2.017 \\
\hline 8.00 & $3.268 \times 10^{5}$ & $3.256 \times 10^{3}$ & $3.231 \times 10^{2}$ & $3.189 \times 10^{1}$ & 3.415 \\
\hline
\end{tabular}




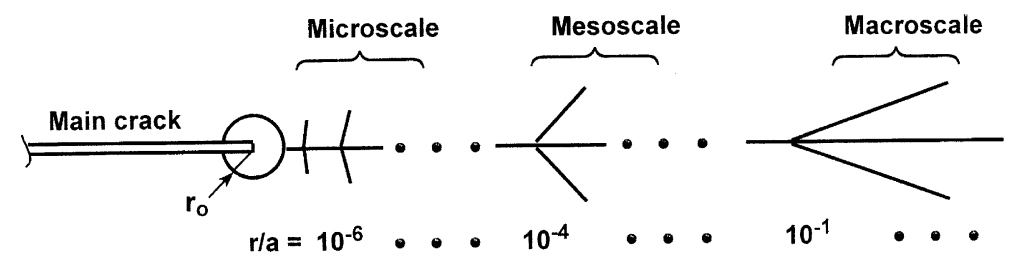

Fig. 3. Distance dependant character of bifurcation damage for PZT-4 with $v=1300 \mathrm{~m} / \mathrm{s}$ and $E / \sigma=-0.0533$.

$E_{y}^{\infty} / \sigma_{y}^{\infty}=-0.0266$ and 0.00 , minima of $\mathrm{d} W / \mathrm{d} V$ were not found and hence crack initiation direction(s) are not reported.

Physical interpretation of the results in Table 4 is illustrated in Fig. 3. Three size and time regimes are shown; they are referred to as micro, meso and macro. In the microscale region, tiny fissures are predicted; they attain very large bifurcation angles of $\pm 72^{\circ}$ and then decrease to $\pm 16.2^{\circ}$ for $E_{y}^{\infty} /$ $\sigma_{y}^{\infty}=-0.0533 \mathrm{Vm} / \mathrm{N}$. For $E_{y}^{\infty} / \sigma_{y}^{\infty}>0$, these fissures tend to coincide with the path of load symmetry. The time available for the creation of these fissures are very short and hence their sizes are expected to be small. Increase of the branch sizes are expected to occur in the mesoscale range with decreasing angles of inclination. These effects are most pronounced for bifurcation at the macroscale where the angles are reduced to $\pm 16.2^{\circ}$ and the branches would be longer as more time is available to create free surface.

At each scale, $(\mathrm{d} W / \mathrm{d} V)_{\min }$ would have to exceed a threshold for the nucleation of a straight or forked crack, i.e., $(\mathrm{d} W / \mathrm{d} V)_{\min }>(\mathrm{d} W / \mathrm{d} V)_{\mathrm{c}}$. It is not difficult to anticipate that

$$
\left(\frac{\mathrm{d} W}{\mathrm{~d} V}\right)_{\mathrm{c}}^{\text {micro }}>\left(\frac{\mathrm{d} W}{\mathrm{~d} V}\right)_{\mathrm{c}}^{\text {meso }}>\left(\frac{\mathrm{d} W}{\mathrm{~d} V}\right)_{\mathrm{c}}^{\text {macro }}
$$

If $(\mathrm{d} W / \mathrm{d} V)^{\text {macro }}$ in Eq. (27) is in MPa, then $(\mathrm{d} W /$ $\mathrm{d} V)^{\text {micro }}$ would be in GPa. Their precise values would depend on the material under consideration.

\section{Moving crack inertia}

A crack is said to carry inertia as it encounters a material particle in or near its path. Increase of the particle velocity would invariably increase the kinetic energy density $\mathrm{d} K / \mathrm{d} V$. Since the local stress and strain field would also increase, the strain energy density $\mathrm{d} W / \mathrm{d} V$ also tends to be elevated. These two energy density changes depend on crack velocity and material properties. A relative comparison could provide information on material inertia that a moving crack would have to overcome in order to attain a certain velocity. This is expected to be distance dependent since both $\mathrm{d} K / \mathrm{d} V$ and $\mathrm{d} W / \mathrm{d} V$ are expected to decay with distance from the tip.

\subsection{Low electromechanical coupling: PZT-6B}

A second material PZT-6B ceramic is depicted to examine the changes caused by different material properties. Refer to Table 6 for the piezoelastic constants of this material. Compared with Table 1 for PZT-4, the piezoelectric constant for PZT-6B is lower on the average by a factor of 3 .

Both the kinetic energy density $\mathrm{d} K / \mathrm{d} V$ and strain energy density $\mathrm{d} W / \mathrm{d} V$ decrease rapidly with $r / a$. Plotted in Fig. 4(a) and (b) are, respectively, the ratio $[(\mathrm{d} K / \mathrm{d} V) /(\mathrm{d} W / \mathrm{d} V)] / v^{2}$ versus logarithm of $r / a$ for $E_{y}^{\infty} / \sigma_{y}^{\infty}=-0.08,0.0$, and $0.16 \mathrm{~V} \mathrm{~m} / \mathrm{N}$ and different crack velocities from 10 to $2100 \mathrm{~m} / \mathrm{s}$.

Table 6

Material constants for PZT-6B piezoceramic

\begin{tabular}{|c|c|c|c|c|c|c|c|c|}
\hline \multicolumn{4}{|c|}{ Elastic constants $\times 10^{10}\left(\mathrm{~N} / \mathrm{m}^{2}\right)$} & \multicolumn{3}{|c|}{ Piezoelectric constants $\left(\mathrm{C} / \mathrm{m}^{2}\right)$} & \multicolumn{2}{|c|}{$\begin{array}{l}\text { Dielectric } \\
\text { permittivities } \times 10^{-9}(\mathrm{C} / \mathrm{V} \mathrm{m})\end{array}$} \\
\hline$c_{11}$ & $c_{13}$ & $c_{33}$ & $c_{44}$ & $e_{31}$ & $e_{33}$ & $e_{15}$ & $\varepsilon_{11}$ & $\varepsilon_{33}$ \\
\hline 16.8 & 6.0 & 16.3 & 2.71 & -0.9 & 7.1 & 4.6 & 3.6 & 3.4 \\
\hline
\end{tabular}




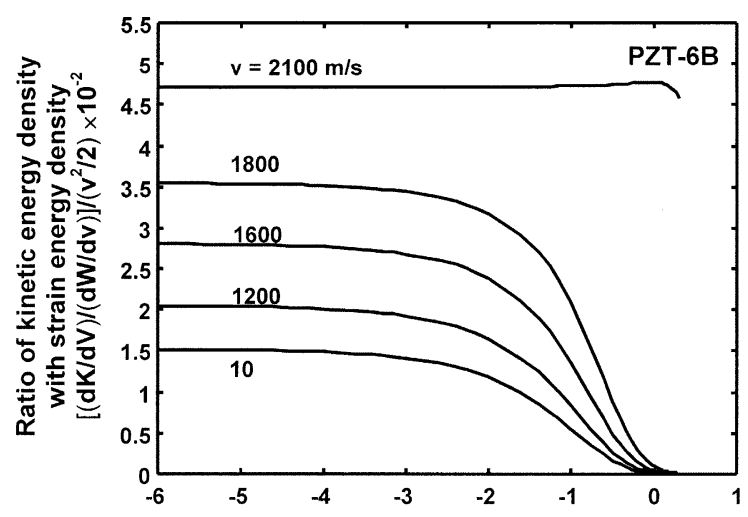

(a)

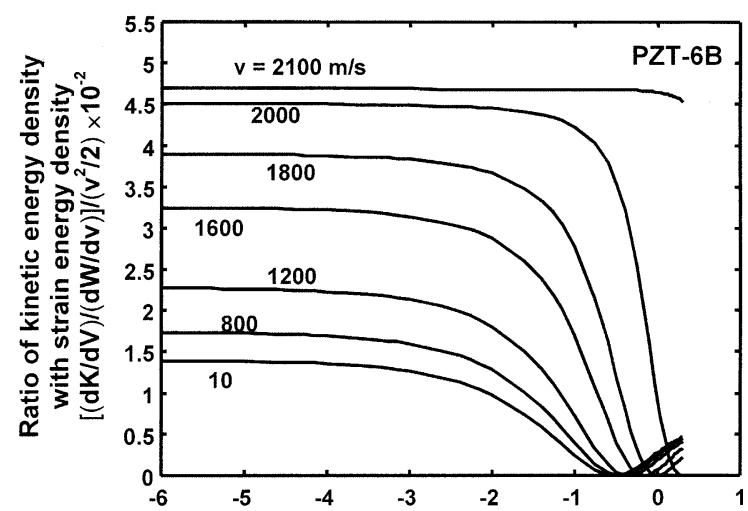

(b)

Logarithm of distance r/a

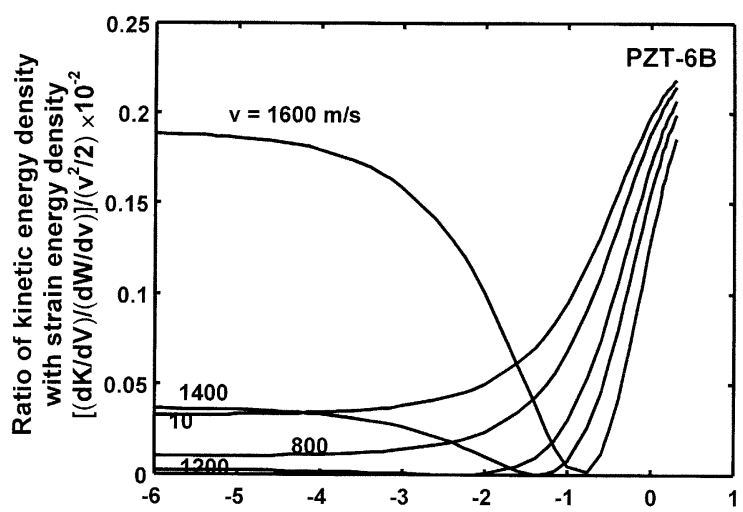

(c)

Logarithm of distance r/a

Fig. 4. Crack-carry inertia for PZT-6B with different velocities and $E_{y}^{\infty} / \sigma_{y}^{\infty}$ ratio. (a) $E_{y}^{\infty} / \sigma_{y}^{\infty}=-0.08 \mathrm{Vm} / \mathrm{N}$, (b) $0.0 \mathrm{Vm} / \mathrm{N}$, (c) $0.08 \mathrm{Vm} / \mathrm{N}$.
For $E_{y}^{\infty} / \sigma_{y}^{\infty}=-0.08 \mathrm{Vm} / \mathrm{N}$ in Fig. 4(a), the ratio remains virtually constant for $v=2100 \mathrm{~m} / \mathrm{s}$, i.e., the inertial effect is distant independent. For $v<$ $1600 \mathrm{~m} / \mathrm{s}$, the curves tend to drop as the distance from the crack is increased. This corresponds to $r / a=10^{-2}$, beyond which all curves tend to zero. The drop is more pronounced for the higher crack velocity curve. No essential changes take place when $E_{y}^{\infty} / \sigma_{y}^{\infty}$ is increased to 0.0 except that the tail ends of the curves start to rise as $r / a \rightarrow 1.0$. This behavior becomes more evident for $E_{y}^{\infty} / \sigma_{y}^{\infty}=0.16$ $\mathrm{Vm} / \mathrm{N}$ and $v<1600 \mathrm{~m} / \mathrm{s}$ in Fig. 4(c). Curvature reversal does not occur for $v>2000 \mathrm{~m} / \mathrm{s}$. This is caused by the non-vanishing strain at infinity where the material particles would experience a velocity in the $x$-direction:

$\dot{u}_{x}=-v \frac{\partial u_{x}}{\partial \xi}=-v \in_{x} \quad$ or $\quad \dot{u}_{x}^{\infty}=-v \in_{x}^{\infty}$

The kinetic energy density will thus be finite regardless of how much $r / a$ is increased unless an opposing strain field is introduced to negate that in Eq. (28). In this way, $\mathrm{d} K / \mathrm{d} V$ would vanish monotonically with distance.

\subsection{High electromechanical coupling: PZT-4}

For the PZT-4 material, Fig. 5(a) for $E_{y}^{\infty} / \sigma_{y}^{\infty}=$ $-0.08 \mathrm{~V} \mathrm{~m} / \mathrm{N}$ shows that the crack inertial effects are larger at a given velocity. They also appear to remain constant for a greater distance before decreasing in magnitude. Similar trends are found in Fig. 5(b) and (c) for $E_{y}^{\infty} / \sigma_{y}^{\infty}=0.0$ and $0.08 \mathrm{~V} \mathrm{~m} / \mathrm{N}$, respectively. Uprising of the curves at $r / a=10^{-1}$ and $10^{\circ}$ is considerably more pronounced at $E_{y}^{\infty} /$ $\sigma_{y}^{\infty}=0.0 \mathrm{~V} \mathrm{~m} / \mathrm{N}$ for PZT-4 in Fig. 5(b) as compared with that in Fig. 4(b) for PZT-6B. The high electromechanical coupling of PZT-4 could be responsible for this. The same applies to the result in Fig. 5(c) even though the applied electric field for PZT-4 is only one-half of the strength of that for PZT-6B in Fig. 4(c).

\section{Bifurcation crack speeds and angles}

The strain energy density criterion [12-14] assumes that failure of material elements occur at 


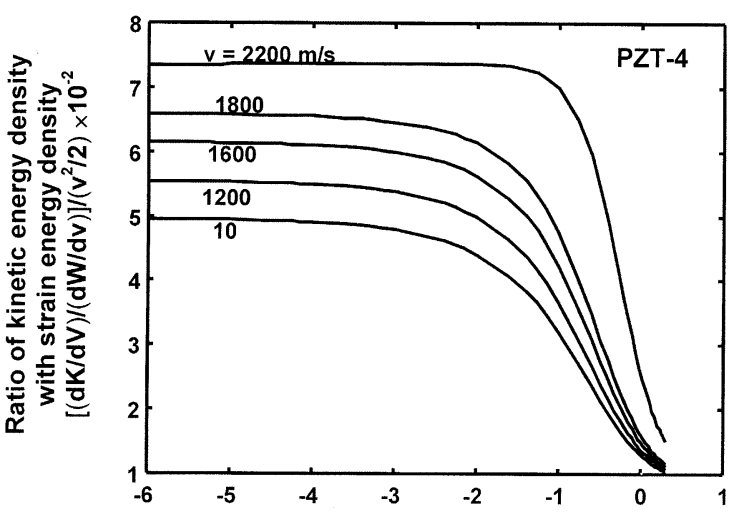

(a)

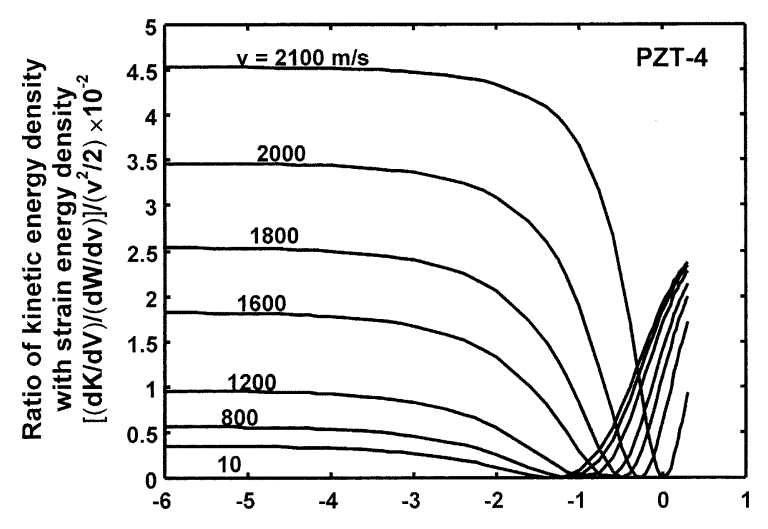

(b)

Logarithm of distance r/a

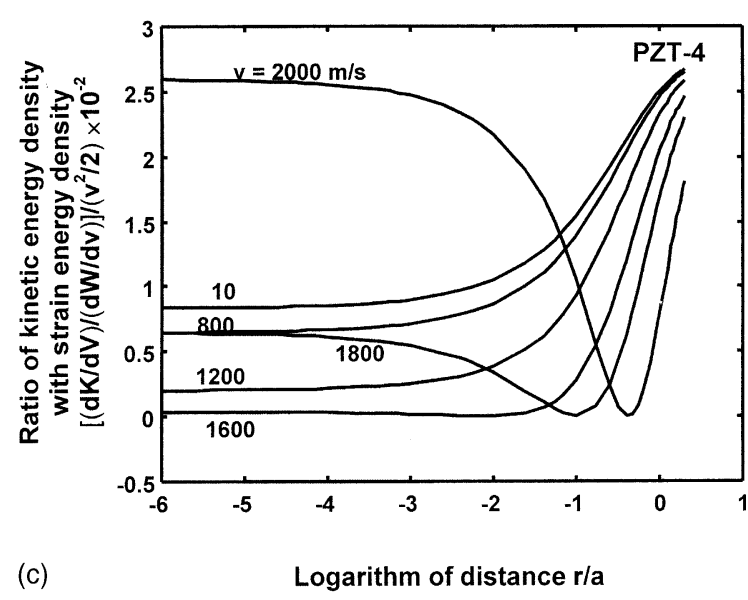

Fig. 5. Crack-carry inertia for PZT-4 with different velocities and $E_{y}^{\infty} / \sigma_{y}^{\infty}$ ratio. (a) $E_{y}^{\infty} / \sigma_{y}^{\infty}=-0.08 \mathrm{Vm} / \mathrm{N}$, (b) $0.0 \mathrm{Vm} / \mathrm{N}$, (c) $0.08 \mathrm{Vm} / \mathrm{N}$. finite distances from the crack tip which is excluded from the analysis by a core region (Fig. 2). Crack propagation is simulated as the coalescence of discrete elements, each of which has exceeded their thresholds represented by the critical volume energy density $(\mathrm{d} W / \mathrm{d} V)_{\mathrm{c}}$. Bifurcation is assumed to initiate when two elements are predicted to fail simultaneously at $\pm \theta_{0}$ off the main course of crack propagation (Fig. 2(b)). Such a model differs from the energy release rate approach where the crack is assumed to cut into the material ahead in a continuous fashion, a mathematical abstraction.

What should be kept in mind is the multiscale implications of the following numerical results as the distance scale for $r / a$ extends from $10^{-6}$ (micro) to $10^{-4}$ (meso) and $10^{-1}$ (macro) (Fig. 3). Since time is inherently coupled with length, the time available for creating free surfaces at the different scale should be kept in mind. The nonequilibrium theory of isoenergy density $[19,20]$ addresses this phenomenon.

\subsection{Distance dependency}

Prior to the arrival of a moving crack, the material particles ahead is disturbed dynamically. Severity of the disturbance would decrease with distance. The material microstructure behavior will be reflected by the macroscopic constants in Table 1 for PZT-4 and Table 6 for PZT-6B. Bifurcation characteristics can be greatly affected. This will be exhibited for $E_{y}^{\infty} / \sigma_{y}^{\infty}=-0.08,0.0$ and $0.08 \mathrm{~V} \mathrm{~m} / \mathrm{N}$.

PZT-6B ceramic: Fig. 6(a)-(c) show, respectively, the variations of bifurcation angles $\pm \theta_{0}$ with distance $r / a$ as $E_{y}^{\infty} / \sigma_{y}^{\infty}$ is altered. The angles increased to about $\pm 70^{\circ}$ for crack velocities of 100 , $900, \ldots, 2000 \mathrm{~m} / \mathrm{s}$ as indicated in Fig. 6(a) for $E_{y}^{\infty} / \sigma_{y}^{\infty}=-0.08 \mathrm{Vm} / \mathrm{N}$. All curves remained flat and then start to drop at about $r / a=10^{-2}$. Bifurcation ceases for large distances away from the tip. This feature is retained in Fig. 6(b) for $E_{y}^{\infty} /$ $\sigma_{y}^{\infty}=0.0 \mathrm{~V} \mathrm{~m} / \mathrm{N}$ except that the curves for different crack velocities are more wide spread. Changes in the bifurcation angles with distance $r / a$ are much more sensitive. This trend continues for $E_{y}^{\infty} / \sigma_{y}^{\infty}=$ $0.08 \mathrm{~V} \mathrm{~m} / \mathrm{N}$ in Fig. 6(c). Now, the velocity curves are further compressed where $v=100$ to $1200 \mathrm{~m} / \mathrm{s}$ 


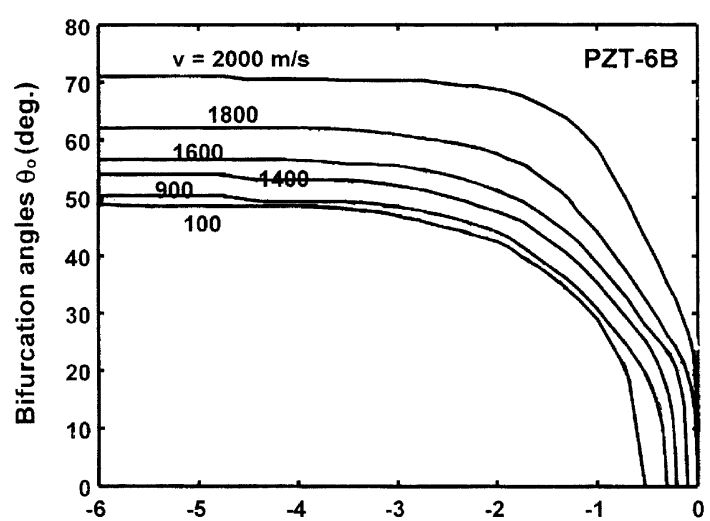

(a)

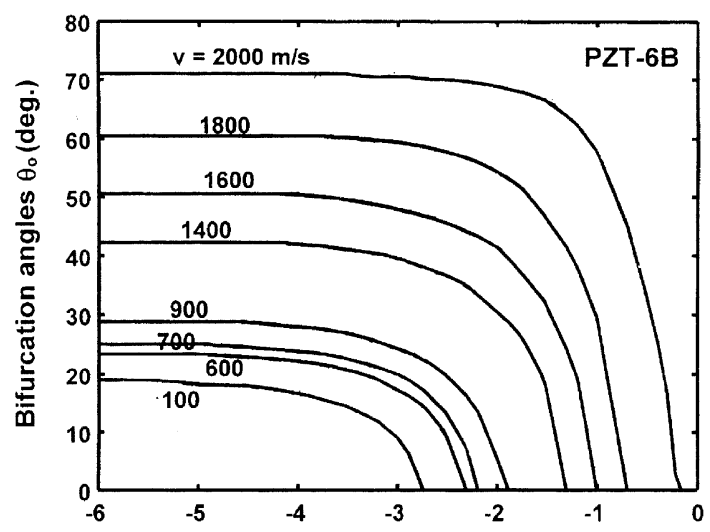

(b)

Logarithm of distance r/a

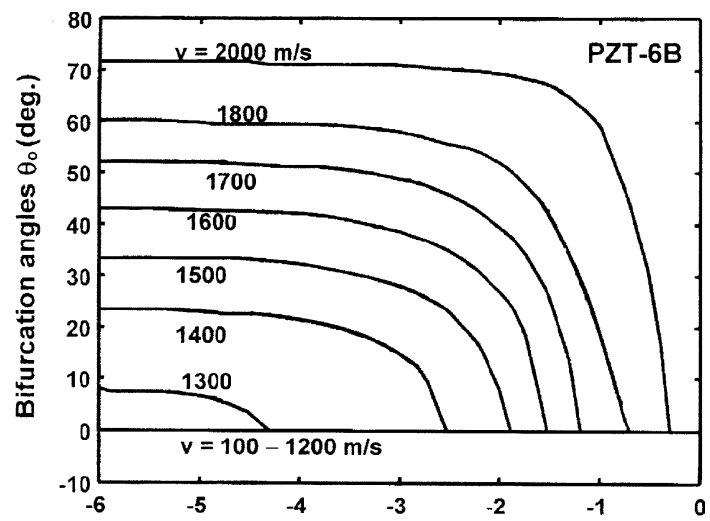

(c)

\section{Logarithm of distance r/a}

Fig. 6. Variations of bifurcation angles with distance for PZT6B with different velocities and $E_{y}^{\infty} / \sigma_{y}^{\infty}$ ratio. (a) $E_{y}^{\infty} / \sigma_{y}^{\infty}=$ $-0.08 \mathrm{~V} \mathrm{~m} / \mathrm{N}$, (b) $0.0 \mathrm{Vm} / \mathrm{N}$, (c) $0.08 \mathrm{~V} \mathrm{~m} / \mathrm{N}$. are represented by the line $\theta_{0}=0^{\circ}$ for a single crack path.

PZT-4 ceramic: By keeping all field parameters constant, the material is now changed from PZT6B to PZT-4. A comparison of Fig. 7(a) with Fig. 6(a) to shows that dramatic changes of $\pm \theta_{0}$ versus $\log (r / a)$ have taken place. All the curves in Fig. 7 (a) are now closely confined to $\theta_{0} \approx \pm 70^{\circ}$; they begin to diverge as $r / a \rightarrow 10^{-2}$. Opposing effects are also seen from the results in Fig. 7(b) and (c) when compared with those in Fig. 6(b) and (c) for PZT-6B. The wide spread feature of the crack velocity curves are now replaced by converging trends in Fig. 7(b) and (c). The bifurcation angles no longer change appreciably with distance until $r / a$ reaches about $10^{-2}$.

\subsection{Crack speed changes}

At a fixed distance ahead of the tip, the bifurcation angles would increase with crack speed. This effect becomes less sensitive as the distance $r / a$ is increased. The behavior for PZT-4 and PZT$6 \mathrm{~B}$, however, differs significantly and will be displayed graphically.

PZT-6B ceramic: It can be seen from the curves of $r / a=0.5$ to 1.0 in Fig. 8(a) that relatively small change in $v$ can cause large variations of $\theta_{0}$. Larger change in crack velocity is required to vary the bifurcation angle once $r / a$ becomes smaller than 0.2 . As the ratio $E_{y}^{\infty} / \sigma_{y}^{\infty}$ is increases from -0.08 to $0.00 \mathrm{Vm} / \mathrm{N}$ in Fig. $8(\mathrm{~b})$, change in $\theta_{0}$ becomes more sensitive to crack speed. This is evidenced by the increase of $d \theta_{0} / \mathrm{d} v$, the slope of the curves. Even more dramatic are the results in Fig. 8(c) for $E_{y}^{\infty} / \sigma_{y}^{\infty}=0.08 \mathrm{~V} \mathrm{~m} / \mathrm{N}$ when the curves are nearly vertical for all $r / a$ and they are bunched together in the range of $1300-2000 \mathrm{~m} / \mathrm{s}$.

PZT-4 ceramic: Illustrated in Fig. 9(a)-(c) are similar results for PZT-4. Very close to the tip, little or no changes could be seen in $\theta_{0}$ for the range of crack velocities considered. This is shown in Fig. 9(a) for $E_{y}^{\infty} / \sigma_{y}^{\infty}=-0.08 \mathrm{~V} \mathrm{~m} / \mathrm{N}$. Beyond the distance $r / a=10^{-2}$, the bifurcation angle decreases with $v$ and then rises. This is opposite to the behavior of PZT-6B in Fig. 8(a) for $v$ within the interval of $1150-2180 \mathrm{~m} / \mathrm{s}$. Decreasing in $v$ is shifted to the curves for $r / a=10^{-6}$ and $10^{-3}$ in 


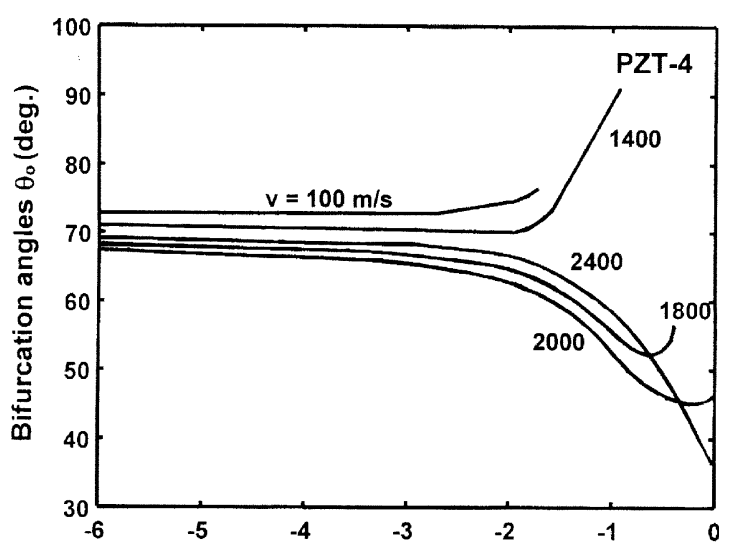

(a)
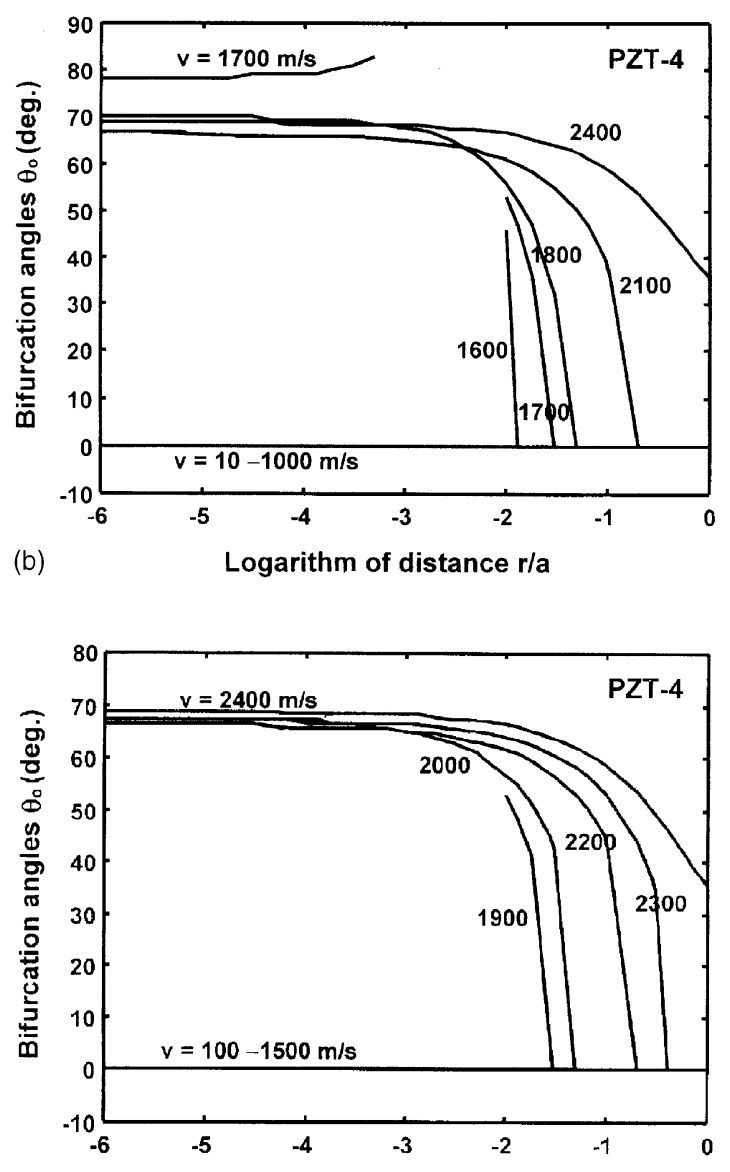

(c)

Logarithm of distance r/a

Fig. 7. Variations of bifurcation angles with distance for PZT-4 with different velocities and $E_{y}^{\infty} / \sigma_{y}^{\infty}$ ratio. (a) $E_{y}^{\infty} / \sigma_{y}^{\infty}=-0.08$ $\mathrm{Vm} / \mathrm{N}$, (b) $0.0 \mathrm{Vm} / \mathrm{N}$, (c) $0.08 \mathrm{~V} \mathrm{~m} / \mathrm{N}$.
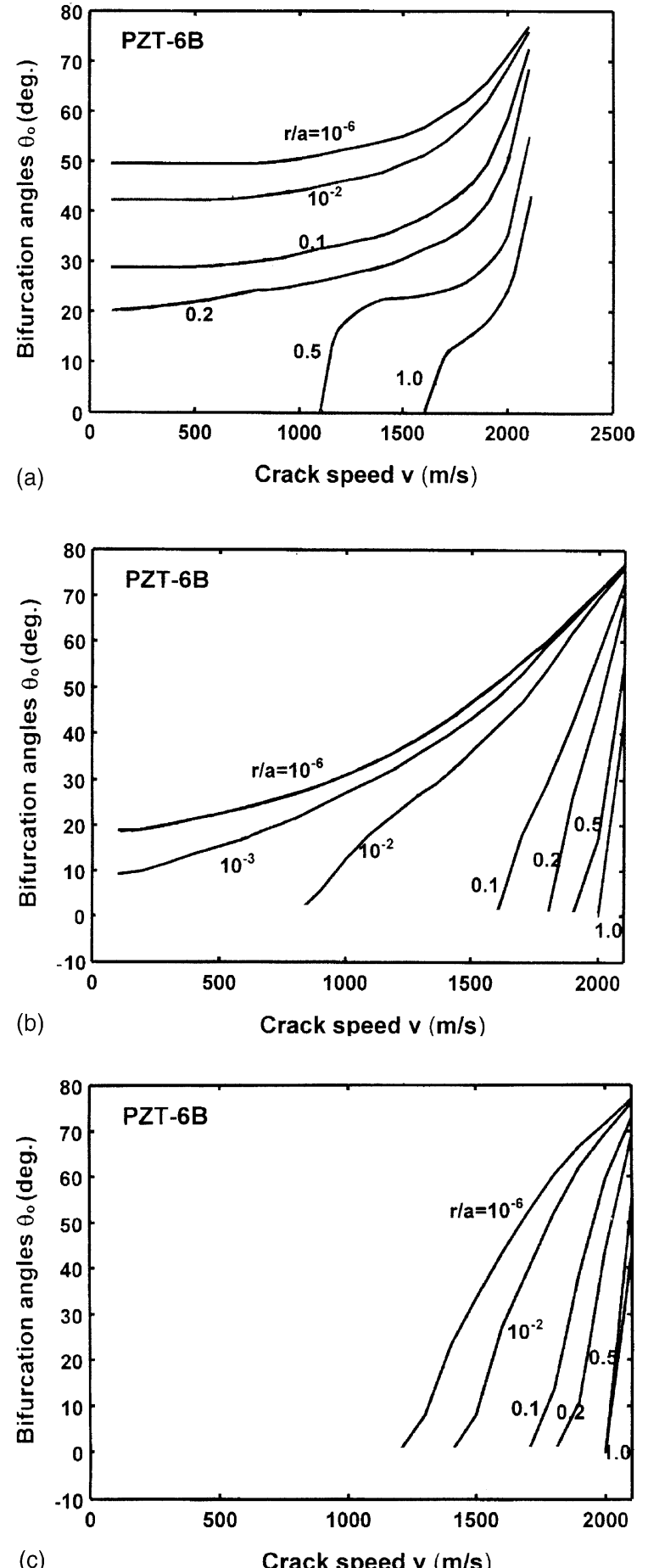

Fig. 8. Bifurcation angles against crack speed for PZT-6B with different distances and $E_{y}^{\infty} / \sigma_{y}^{\infty}$ ratio. (a) $E_{y}^{\infty} / \sigma_{y}^{\infty}=-0.08 \mathrm{Vm} /$ $\mathrm{N}$, (b) $0.0 \mathrm{~V} \mathrm{~m} / \mathrm{N}$, (c) $0.08 \mathrm{Vm} / \mathrm{N}$. 


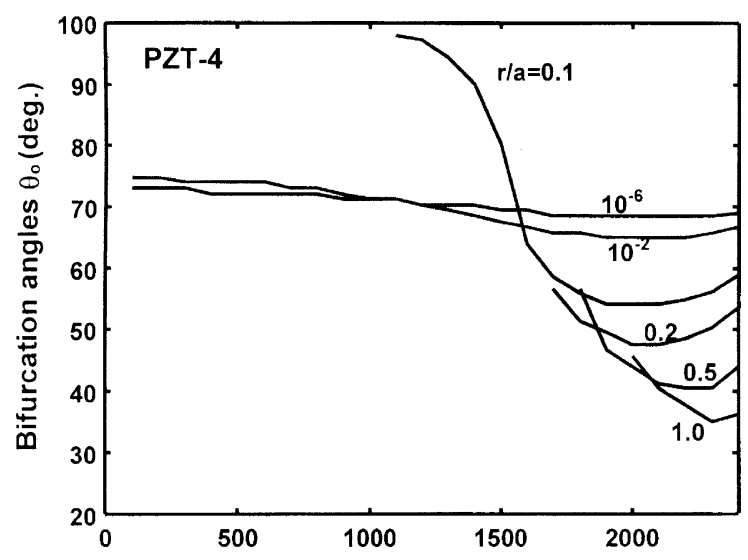

(a)
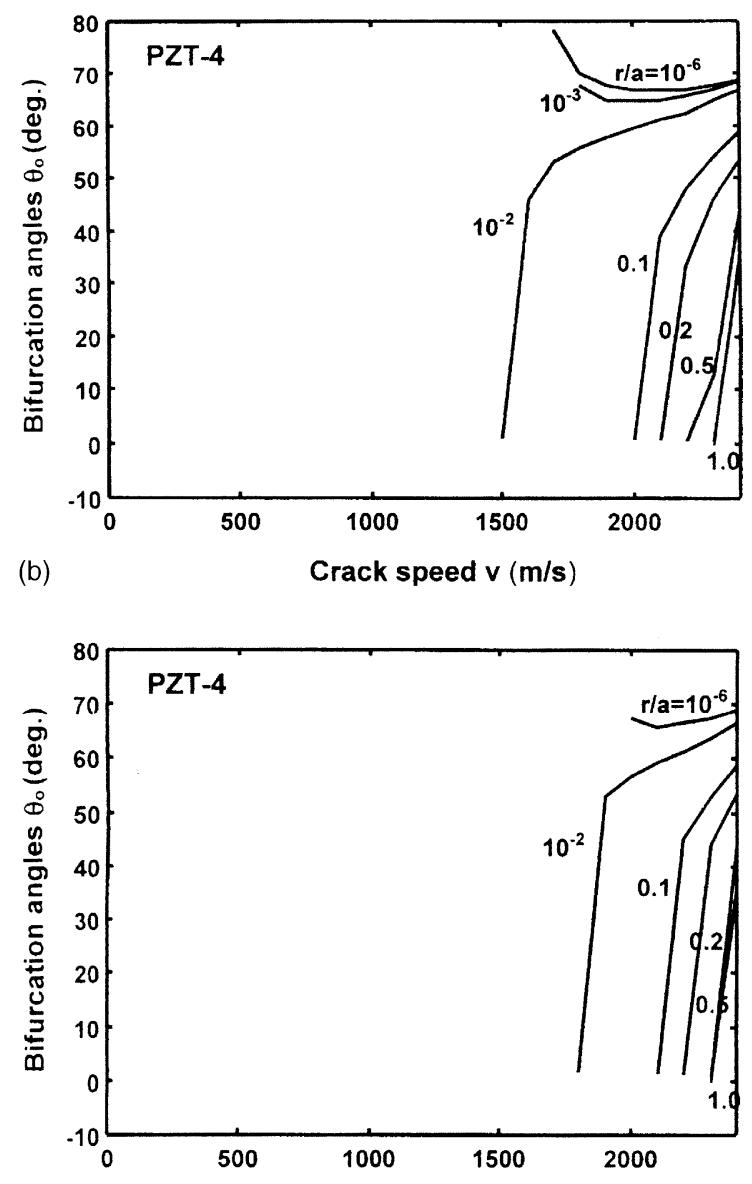

(c)

Crack speed $v(\mathrm{~m} / \mathrm{s})$

Fig. 9. Bifurcation angles against crack speed for PZT-4 with different distances and $E_{y}^{\infty} / \sigma_{y}^{\infty}$ ratio. (a) $E_{y}^{\infty} / \sigma_{y}^{\infty}=-0.08 \mathrm{~V} \mathrm{~m} /$ $\mathrm{N}$, (b) $0.0 \mathrm{~V} \mathrm{~m} / \mathrm{N}$, (c) $0.08 \mathrm{~V} \mathrm{~m} / \mathrm{N}$.
Fig. 9(b) as $E_{y}^{\infty} / \sigma_{y}^{\infty}$ is increased to 0.00 while large changes in $\theta_{0}$ are seen for those curves with $r / a>10^{-2}$ as they adopt a steep descent. Fig. 9(c) for $E_{y}^{\infty} / \sigma_{y}^{\infty}=0.08 \mathrm{Vm} / \mathrm{N}$ shows that a slight decrease of $\theta_{0}$ is still retained in the curve for $r / a=10^{-6}$ but the remaining curve drop even more rapidly for $v>1780 \mathrm{~m} / \mathrm{s}$.

\subsection{Effect of poling and electric field strength}

The electric field strength and its direction with reference to poling can also influence the bifurcation angle at different crack velocities. In view of the variance in results for the two ceramics, it is expected that similar differences will also be observed in this case.

PZT-6B ceramic: In Fig. 10(a)-(c), the crack velocities are varied from 100 to $2000 \mathrm{~m} / \mathrm{s}$ while $\theta_{0}$ is plotted against $E_{y}^{\infty} / \sigma_{y}^{\infty}$ for fixed $r / a=10^{-6}, 10^{-2}$ and $10^{-1}$. The high velocity curve $(v=2000 \mathrm{~m} / \mathrm{s})$ remained unchanged for the range of $E_{y}^{\infty} / \sigma_{y}^{\infty}$ considered. The onset of crack bifurcation $\theta_{0}>0^{\circ}$ corresponds to positive $E_{y}^{\infty}$ and shifts gradually into the negative $E_{y}^{\infty}$ regime as $r / a$ is increased to $10^{-1}$. The curves are well behaved. They reveal that the bifurcation angles are confined within $\pm 29^{\circ}$ to $\pm 58^{\circ}$ for $E_{y}^{\infty} / \sigma_{y}^{\infty}=-0.08 \mathrm{~V} \mathrm{~m} / \mathrm{N}$.

PZT-4 ceramic: Compared with the results in Fig. 10(a) and (b) for PZT-6B, the curves for PZT4 in Fig. 11(a) with $v \leqslant 1800 \mathrm{~m} / \mathrm{s}$ and in Fig. 11(b) with $v \leqslant 1400 \mathrm{~m} / \mathrm{s}$ acquired an opposite behavior. They correspond to an increase in $\theta_{0}$ with decreasing $E_{y}^{\infty} / \sigma_{y}^{\infty}$. Similar feature is observed as $r / a$ is increased to $10^{-1}$ in Fig. 11(c). No bifurcation has been found for $v=100$ to $900 \mathrm{~m} / \mathrm{s}$ and full range of $E_{y}^{\infty} / \sigma_{y}^{\infty}$ shown.

\subsection{Strain energy density variations}

Assuming that the process of free surface creation is irreversible, a system could not gain energy by self-sealing as this would imply a negative energy release rate, the physical meaning of which would be trying [21]. The strain energy density function is required to be positive definite under all conditions. This will be shown numerically for $\mathrm{d} W / \mathrm{d} V$ in Eq. (26). Also keep in mind is that each point on a given $\mathrm{d} W / \mathrm{d} V$ curve corresponds to a 


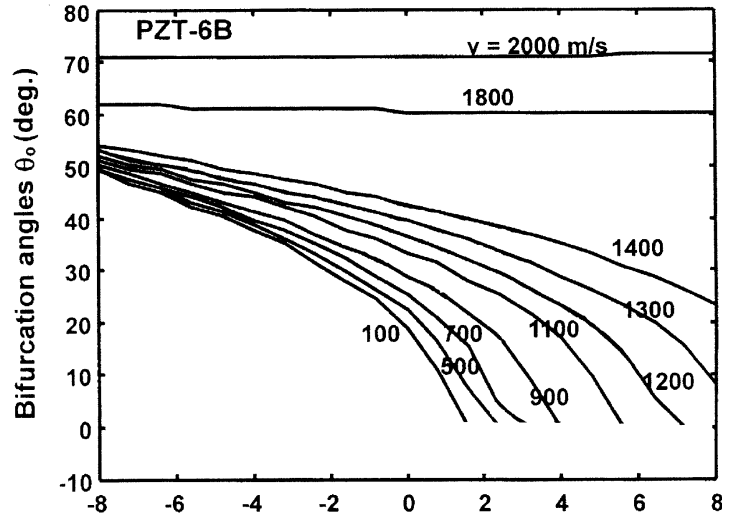

(a) Electric field to stress ratio $E_{y}^{\infty} / \sigma_{y}^{\infty} \times 10^{-2}$

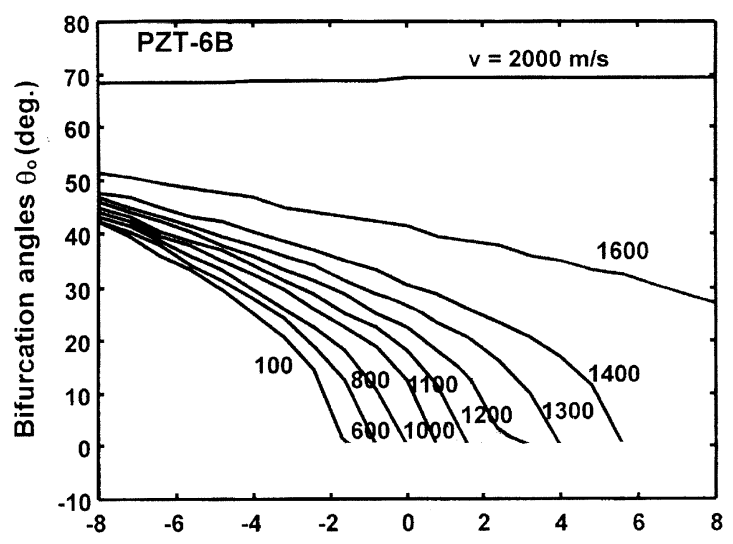

(b) Electric field to stress ratio $E_{y}^{\infty} / \sigma_{y}^{\infty} \times 10^{-2}$

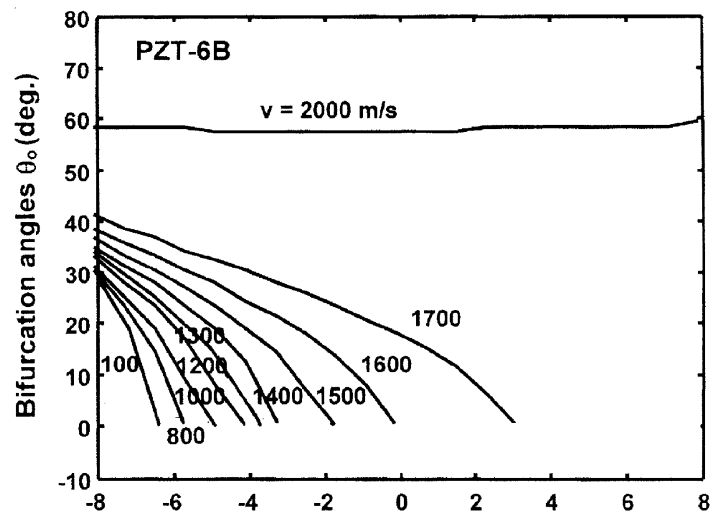

(c) Electric field to stress ratio $E_{y}^{\infty} / \sigma_{y}^{\infty} \times 10^{-2}$

Fig. 10. Variations of bifurcation angles with electric field to stress ratio for PZT-6B with different velocities and $r / a$ ratio. (a) $r / a=10^{-6}$, (b) $10^{-2}$, (c) $10^{-1}$.

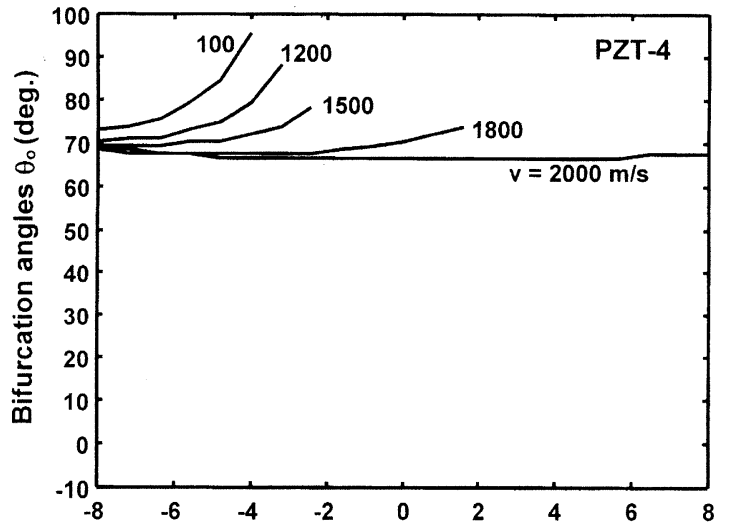

(a) Electric field to stress ratio $E_{y}^{\infty} / \sigma_{y}^{\infty} \times 10^{-2}$

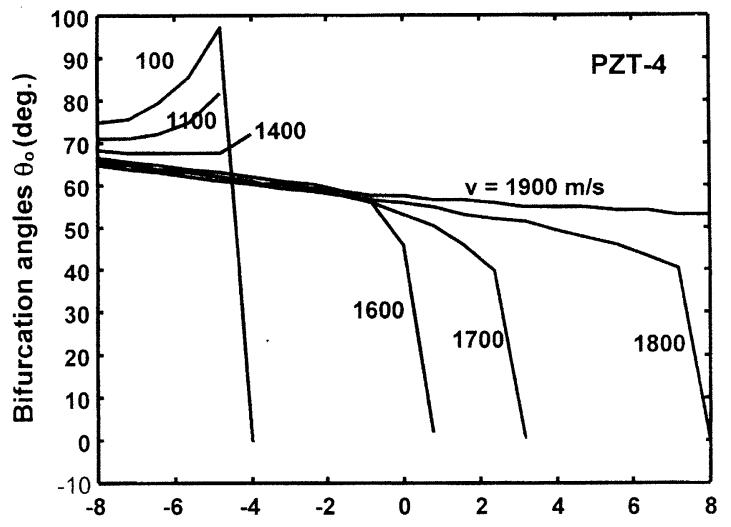

(b) Electric field to stress ratio $E_{y}^{\infty} / \sigma_{y}^{\infty} \times 10^{-2}$

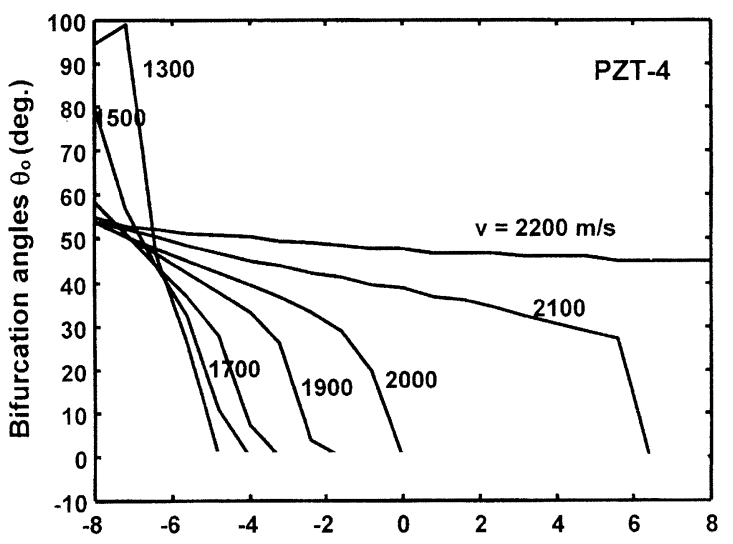

(c) Electric field to stress ratio $E_{y}^{\infty} / \sigma_{y}^{\infty} \times 10^{-2}$

Fig. 11. Variations of bifurcation angles with electric field to stress ratio for PZT-4 with different velocities and $r / a$ ratio. (a) $r / a=10^{-6}$, (b) $10^{-2}$, (c) $10^{-1}$. 


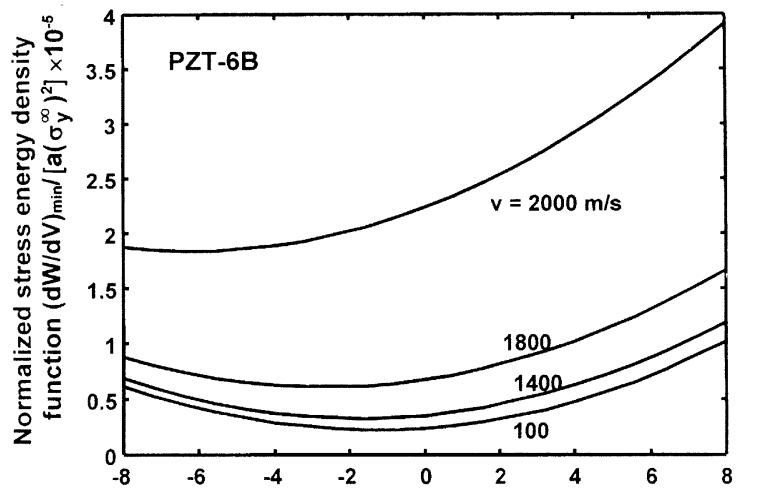

(a)

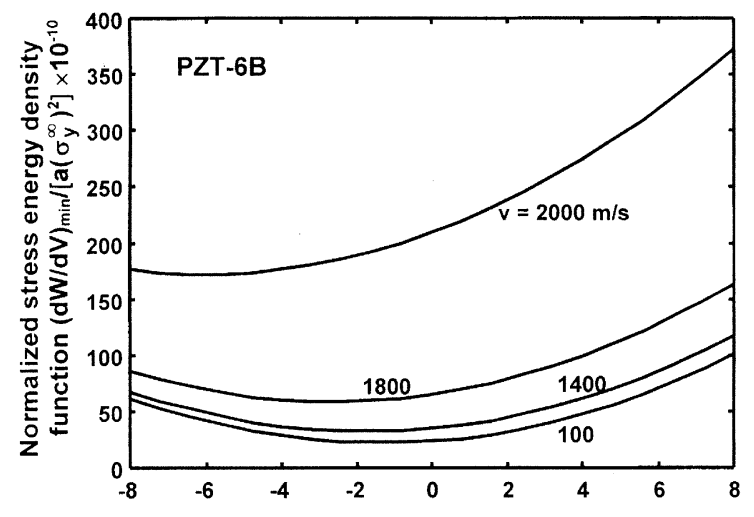

(b) Electric field to stress ratio $E_{y}^{\infty} / \sigma_{y}^{\infty} \times 10^{-2}$

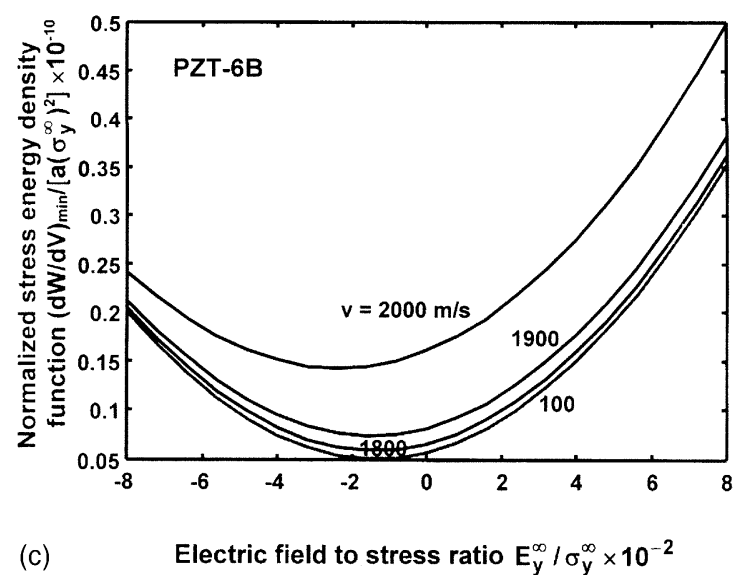

Fig. 12. Normalized strain energy density function versus electric field to stress ratio for PZT-6B with different velocities and distance from tip. (a) $r / a=10^{-6}$, (b) $10^{-3}$, (c) 0.5 . possible energy state of instability where two inclined branches might appear suddenly.

PZT-6B ceramic: A graphical representation of the normalized strain energy density function versus $E_{y}^{\infty} / \sigma_{y}^{\infty}$ is given in Fig. 12(a)-(c) for $r / a=$ $10^{-6}, 10^{-3}$ and $5 \times 10^{-1}$, respectively. Amplitudes of the $\mathrm{d} W / \mathrm{d} V$ curves in Fig. 12(a) are three and six orders of magnitude larger than those in Fig. 12(b) and (c). They could be described by the inequality in Eq. (27) where reference is made to the micro-, meso- and macroscale. The curves are also seen to dip more sharply as $r / a$ is increased to $5 \times 10^{-1}$.

PZT-4 ceramic: The normalized $(\mathrm{d} W / \mathrm{d} V)_{\min }$ curves in Fig. 13(a)-(c) for PZT-4 have similar trends. However, they become more closely spaced for negative electric field and widely spaced for positive electric field. Recall that the size scale of $\mathrm{d} W / \mathrm{d} V$ in Fig. 13(a)-(c) can differ by several orders of magnitude. Assignment of $\left|E_{y}^{\infty}\right|$ values should be exercised with application in mind such that coercive strength would not be exceeded to cause possible domain switching. Change in the ceramic microstructure would be a topic beyond the scope of this discussion.

\section{Conclusions and comments}

In elastodynamics, material elements are assumed to transmit disturbances by dilatational and distortional waves at the respective speeds of $c_{\mathrm{d}}$ and $c_{\mathrm{s}}$. Volume change and shape change are thus regarded as the two fundamental mechanisms of energy transmission; their description is made possible only in two-dimensions. If the lateral contraction of a unit cube is one fourth of the axial extension, this gives $c_{\mathrm{d}}=c_{\mathrm{s}}=1.732$ which happens to coincide with the speed ratio for glass. Theoretical formulation of the elastodynamic field theory also imposes limitation on speed because it contains the factors $\sqrt{1-\left(v / c_{\mathrm{s}}\right)^{2}}$ and $\sqrt{1-\left(v / c_{\mathrm{d}}\right)^{2}}$ which are required to be non-zero. Hence, $v<c_{\mathrm{s}}$ sufficies because $c_{\mathrm{d}}>c_{\mathrm{s}}$. The Rayleigh surface wave speed $c_{\mathrm{R}}$ being about $92 \%$ of $c_{\mathrm{s}}$, for $v=0.25$ (Poison's ratio) has been most frequently quoted as a limit even though there is no physical reasons why $c_{\mathrm{d}}$ or $c_{\mathrm{s}}$ should be the upper 


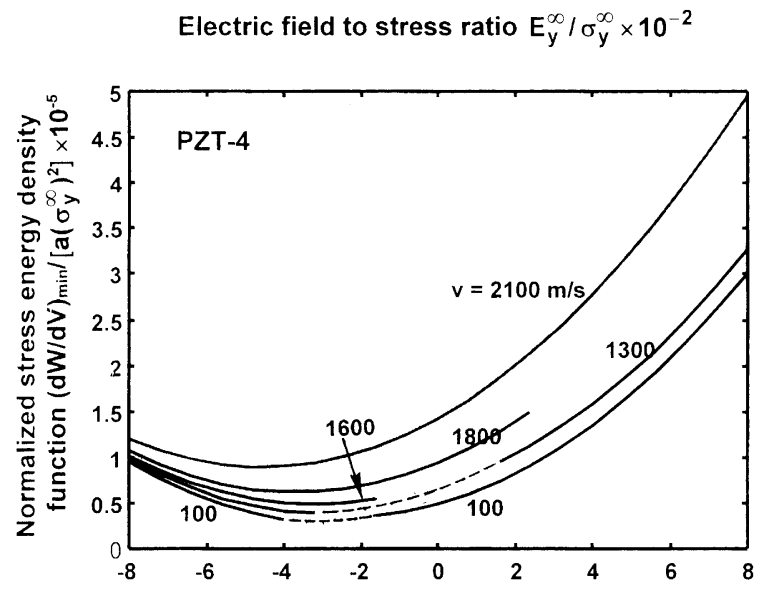

(a)

Electric field to stress ratio $\mathrm{E}_{\mathrm{y}}^{\infty} / \sigma_{\mathrm{y}}^{\infty} \times 10^{-2}$

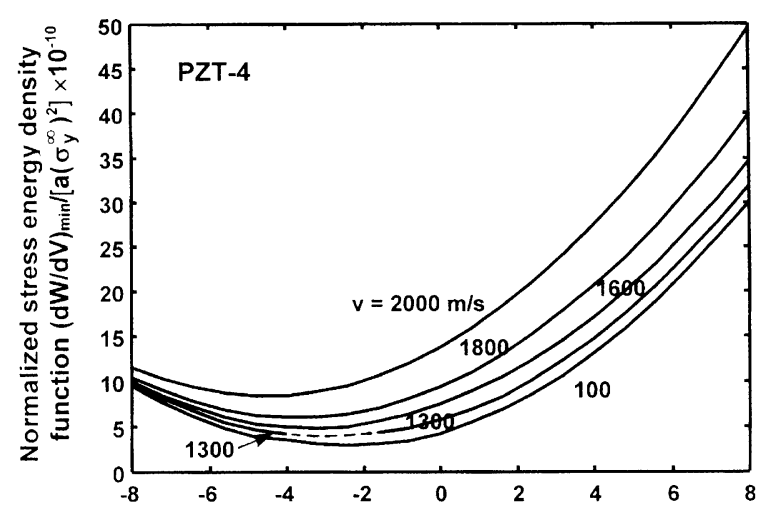

(b)

Electric field to stress ratio $E_{y}^{\infty} / \sigma_{y}^{\infty} \times 10^{-2}$

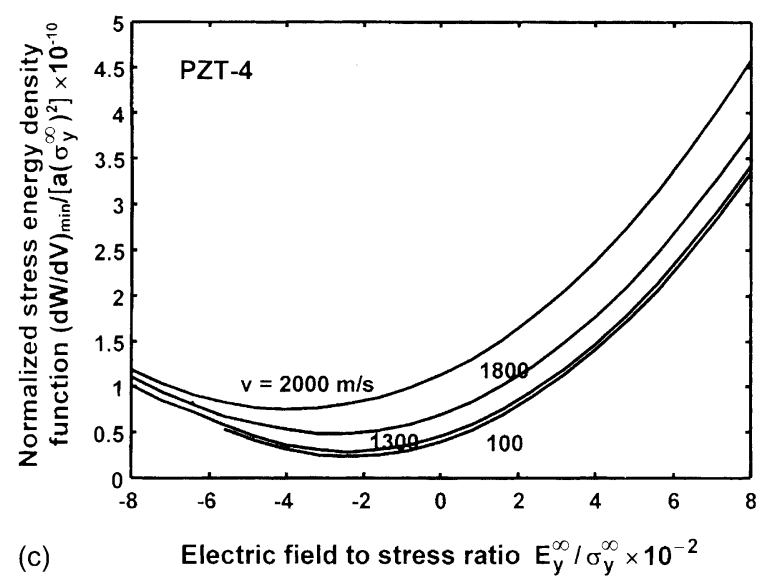

Fig. 13. Normalized strain energy density function versus electric field to stress ratio for PZT-4 with different velocities and distance from tip. (a) $r / a=10^{-6}$, (b) $10^{-2}$, (c) 0.1 . limits. Keep in mind that these speeds are defined in terms of the bulk mass density and material constants referred to the macroscopic scale for an isotropic and homogeneous medium. Violation of these assumptions would render the results unmeaningful.

The classical definitions of $c_{\mathrm{s}}$ and $c_{\mathrm{d}}$ become dubious at the microscopic scale where inhomogeneity takes precedent. When changes are considered at the local scale, the system is said to be in state of "non-equilibrium" $[19,20]$. That is the material properties would alter with the time and may not be represented by the same parameters at different scale levels. Assessment of dilatational and distortional effects at a given size and time scale can be made automatically from the stationary values of the strain energy density function [12-14] even for non-linear situations. Linear superposition is not necessary. Such a procedure has indeed led to a better understanding of how the field characterization parameters control crack bifurcation. The uniform motion crack solution is not able to shed light on the transient character of dividing a single crack path into two. Assuming that the crack attains a constant velocity from the outset is equivalent to sudden bifurcation. Even then, initiation of microfissures, mesocracks and macrobranches are predicted along the prospectively path of crack propagation. They could contribute to the roughness of the fracture surfaces related to branching.

Shown are changes in bifurcation behavior caused by crack velocity, electric field strength and material properties. Different results are obtained for different distances from the crack tip. The display could involve the variations of bifurcation crack speed as a function of the electric field to applied stress ratio. Fig. 14 gives a family of curves with $r / a=10^{-6}, 10^{-3}, \ldots, 10^{0}$ using the PZT-6B material for illustration. For a given $E_{y}^{\infty} / \sigma_{y}^{\infty}$ ratio, the likelihood of a sudden bifurcation can be identified with a distance $r / a$ and crack speed. Bifurcation speed can also be plotted against the distance ratio $r / a$ using $E_{y}^{\infty} / \sigma_{y}^{\infty}$ as the varying parameter (Fig. 15). The curves would then clearly show that the speed at which a crack is predicted to bifurcate tend to remain constant for distances close to the tip if the electric field to stress ratio is 


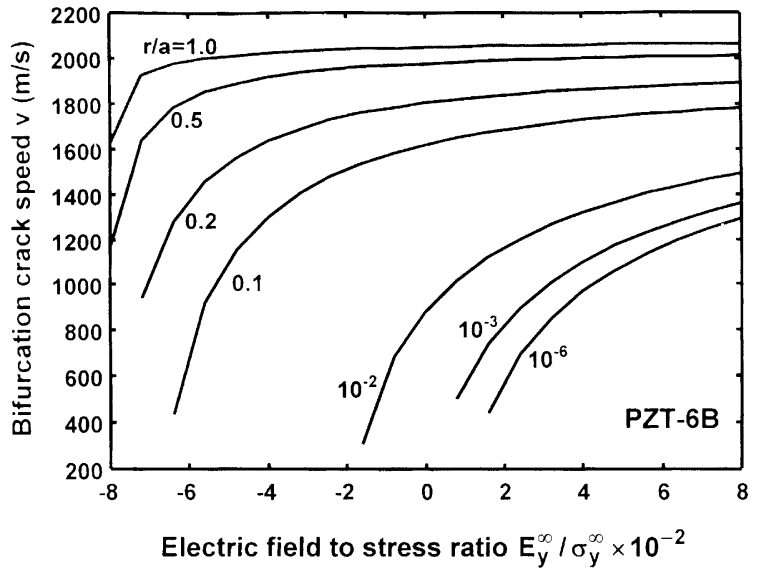

Fig. 14. Crack speed at bifurcation versus electric field to stress ratio for PZT-6B.

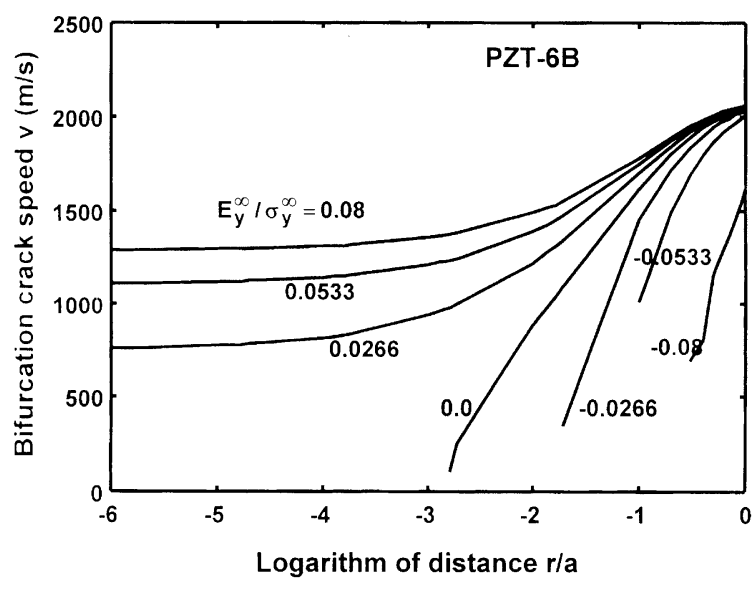

Fig. 15. Crack speed at bifurcation versus distance from tip for PZT-6B.

kept greater than $0.0266 \mathrm{Vm} / \mathrm{N}$. Curves labelled with $E_{y}^{\infty} / \sigma_{y}^{\infty}=-0.08,-0.0533,-0.0266$ and 0.00 $\mathrm{V} \mathrm{m} / \mathrm{N}$ lie in the region for $r / a>10^{-3}$. The coordinates of their ends are $(-0.52,691),(-1.00$, $1011),(-1.71,346)$ and $(-2.79,105)$. In general, a high crack speed would be required for bifurcation to initiate at distances further away from the tip. Graphical representations involving other parameters may be developed in the same way.

An improved understanding of the transient character of bifurcation is handicapped by the assumption where the crack speed is taken to be constant for all time. The results must therefore be assessed accordingly. Although non-uniform crack growth rates could be treated for quasi-static problems, the effective treatment of crack acceleration does not appear to be in sight.

\section{Uncited reference}

[22]

\section{References}

[1] F.F. Abraham, H.J. Gao, How fast can crack propagate, Phys. Rev. Lett. 84 (14) (2000) 3113-3116.

[2] D.J. Andrews, Rupture velocity of plane strain shear cracks, J. Geophys. Res. 81 (32) (1976) 5667-5687.

[3] R. Burridge, G. Conn, L.B. Freund, The stability of a rapid mode II shear crack with finite cohesive traction, J. Geophys. Res. 85 (B5) (1979) 2210-2222.

[4] E.T. Embley, G.C. Sih, Plastic flow around an expanding crack, J. Engng. Fract. Mech. 4 (1972) 431-442.

[5] P.D. Washabaugh, W.G. Knass, A reconciliation of dynamic crack velocity and Rayleigh wave speed in isotropic brittle solid, Int. J. Fract. 65 (2) (1994) 97-114.

[6] A.J. Rosakis, C. Sarnudrala, D. Coker, Crack faster than the shear wave speed, Science 284 (1999) 1337-1340.

[7] Z. Zhu, K. Ravi-Chandar, Experiments on dynamic shear crack propagation, in: Proceedings of the Int'l Conf. on Adv'd Techniques in Experiments, 1999, pp. 395402.

[8] P.A. Klein, J.W. Foulk, E.P. Chen, S.A. Wimmer, H.J. Gao, Physical model of brittle fracture: cohesive formulation and meshfree method, Prospects of mesomechanics in the 21 st century, in: G.C. Sih, V.E. Panin (Eds.), J. Theor. Appl. Fract. Mech. 37 (2002) 99-166.

[9] G.C. Sih, Implication of scaling hierarchy associated with nonequilibrium: field and particulate, Prospects of mesomechanics in the 21st century, in: G.C. Sih, V.E. Panin (Eds.), Theor. Appl. Fract. Mech. 37 (2001) 335-369 (special issue).

[10] G.C. Sih, B. Liu, Meso-fracture mechanics: a necessary link, Prospects of mesomechanics in the 21st century, in: G.C. Sih, V.E. Panin (Eds.), J. Theor. Appl. Fract. Mech. 37 (2002) 371-395.

[11] G.C. Sih, Dynamic crack problems: strain energy density fracture theory, in: Mechanics of Fracture, Elastodynamic Crack Problems, vol. IV, Noordhoff International Publishing, The Netherlands, 1976, pp. XVIII-XLVII.

[12] G.C. Sih, Strain energy density factor applied to mixed model crack problems, Int. J. Fract. Mech. 10 (1974) 305321.

[13] G.C. Sih, Fracture mechanics of engineering structural components, in: G.C. Sih, L. Faria (Eds.), Fracture 
Mechanics Methodology, vol. 1, Martinus Nijhoff Publishers, The Netherlands, 1984 , pp. 35-101.

[14] G.C. Sih, Mechanics of Fracture Initiation and Propagation, Kluwer Academic Publishers, The Netherlands, 1991.

[15] E.H. Yoffe, The moving Griffith crack, Philos. Mag. 42 (1951) 739-750.

[16] G.C. Sih, G.R. Irwin, Dynamic analysis for two-dimensional multiple crack division, J. Engng. Fract. Mech. 1 (4) (1970) 603-614.

[17] G.R. Irwin, G.C. Sih, Dynamic energy release rate expression for a spreading circular fracture pattern in a plate, J. Theor. Appl. Fract. Mech. 5 (1) (1986) 47-50.

[18] G.C. Sih, Z.F. Song, Damage analysis of tetragonal perovskite structure ceramics implicated by asymptotic field solutions and boundary conditions, J. Theor. Appl. Fract. Mech. 38 (2002) 15-36.

[19] G.C. Sih, Thermomechanics of solids: nonequilibrium and irreversibility, J. Theor. Appl. Fract. Mech. 9 (3) (1988) 175-198.

[20] G.C. Sih, Thermomechanics of solids: nonequilibrium and irreversibility, J. Theor. Appl. Fract. Mech. 9 (3) (1988) 175-198.

[21] G.C. Sih, Some basic problems in nonequilibrium and thermomechanics, in: S. Sienietyez, P. Salamon, Tayler and Francis, New York, 1992, pp. 218-247.

[22] Z. Suo, C.M. Kuo, D.M. Barnett, J.R. Willis, Fracture mechanics for piezoelectric ceramics, J. Mech. Phys. Solid. 40 (1992) 739-765. 